Table A5. - Correlation matrix, model C1, clonal site-site correlations were structured to be equal among trials within cycles and equal among trials within pairs of cycles with the same difference in cycle number. The same letter indicates that the correlations were constrained to be equal in the model.

\begin{tabular}{|c|c|c|c|c|c|c|c|c|c|}
\hline & F1011 & F1012 & F1013 & F1106 & F1107 & F1185 & F1186 & F1243 & F1244 \\
\hline F1011 & & 1 & 1 & A & $A$ & B & B & C & C \\
\hline F1012 & & & 1 & A & A & B & B & C & C \\
\hline F1013 & & & & A & A & B & B & C & C \\
\hline F1106 & & & & & 1 & D & D & $E$ & $E$ \\
\hline F1107 & & & & & & D & D & $E$ & $E$ \\
\hline F1185 & & & & & & & 1 & $F$ & $\mathrm{~F}$ \\
\hline F1186 & & & & & & & & $F$ & $\mathrm{~F}$ \\
\hline F1243 & & & & & & & & & 1 \\
\hline F1244 & & & & & & & & & \\
\hline
\end{tabular}

\title{
Provenance Productivity of High and Low Elevation Pinus tecunumanii in Zimbabwe
}

\author{
By B. I. NyokA ${ }^{1)}$, P. Tongoona ${ }^{2)}$ and C. M. Gumbie ${ }^{3)}$
}

(Received $4^{\text {th }}$ June 2010)

\begin{abstract}
The productivity potential of provenances and families of Pinus tecunumanii were determined across a range of environments in four orthogonal field trials in Zimbabwe. Provenances of $P$. tecunumanii from altitudes above $1500 \mathrm{~m}$ (High Elevation) in natural stands in Mexico and Central America were significantly superior $(\mathrm{P}<0.05)$ to provenances from altitudes below $1500 \mathrm{~m}$ (Low Elevation) when planted in Zimbabwe in eightyear mean individual tree volume (1729.2 versus $1588.6 \mathrm{dm}^{3}$ ) at $1760 \mathrm{~m}$ a.s.l.; were not significantly different (1163.1 versus $1143.9 \mathrm{dm}^{3}$ ) at $1450 \mathrm{~m}$ a.s.l.; but the latter was significantly superior (1756.4 versus $\left.1468.6 \mathrm{dm}^{3}\right)$ at $1050 \mathrm{~m}$ a.s.l., and $(720.7$ versus $531.5 \mathrm{dm}^{3}$ ) at $780 \mathrm{~m}$ a.s.l. The most productive provenances were Juquila $\left(26.1 \mathrm{~m}^{3} \mathrm{ha}^{-1} \mathrm{yr}^{-1}\right)$ at $1760 \mathrm{~m}$, Yucul (17.3 $\left.\mathrm{m}^{3} \mathrm{ha}^{-1} \mathrm{yr}^{-1}\right)$ at $1450 \mathrm{~m}$, San Francisco $\left(24.9 \mathrm{~m}^{3} \mathrm{ha}^{-1} \mathrm{yr}^{-1}\right)$ at $1050 \mathrm{~m}$ and Villa Santa $\left(11.1 \mathrm{~m}^{3} \mathrm{ha}^{-1} \mathrm{yr}^{-1}\right)$ at $780 \mathrm{~m}$ a.s.l. Improved $P$. patula was

\footnotetext{
1) Formerly Principal Tree Breeder, Forestry Commission, Forest Research Centre, Harare, Zimbabwe. Current address: World Agroforestry Centre, Chitedze Agricultural Research Station, ICRAF-Southern Africa Programme, P. O. Box 30798, Lilongwe, Malawi. Phone: +265-1-707-329. E-mail: b.nyoka@cgiar.org.

2) Professor Plant Breeding and Genetics, University of KwaZulu-Natal, African Centre for the Crop Improvement, P. Bag X01, Scottsville, Pietermaritzburg 3209, South Africa, E-mail: tongoona@ukzn.ac.za.

3) Biometrician and Deputy General Manager, Forest Research Centre, Forestry Commission, P. O. Box HG595, Highlands, Harare, Zimbabwe. E-mail: cgum@frchigh.co.zw.
}

significantly superior to $P$. tecunumanii at $1760 \mathrm{~m}$ a.s. but the differences were nonsignificant at $1450 \mathrm{~m}$ a.s.l. In the warm lower altitude sites, $P$. tecunumanii was not significantly different from improved $P$. oocarpa, the commonly planted species, although some provenances of $P$. tecunumanii were significantly superior by as much as $31 \%$. The top 10 ranked families of the high elevation $P$. tecunumanii had an eight-year volume advantage of $1 \%$ and $23 \%$ over P. patula at Stapleford and Cashel, and $40 \%$ and $34 \%$ over P. oocarpa at Gungunyana and Maswera respectively. Genotype-environment interaction was significant by elevation group, provenance and family level. The interaction at the family level was however largely contributed by families from the high elevation $P$. tecunumanii. Opportunities exist for immediate deployment of seed of selected provenances of $P$. tecunumanii in medium and low altitude areas to improve plantation productivity. There is however, no immediate yield advantage of using $P$. tecunumanii seed in high potential environments currently planted to $P$. patula. Breeding and selection could also bring about the planting of $P$. tecunumanii in the higher altitudes in the near future.

Key words: Low and High Elevation P. tecunumanii, provenance, productivity.

\section{Introduction}

The Tecun Umán pine, Pinus tecunumanii Eguiluz \& J. P. Perry is a closed-cone pine that occurs from southern Mexico to central Nicaragua in a series of disjunct populations (STYLES and MCCARTER, 1988; DvorAK et al., 
Table 1. - Geographic details of the provenances of $P$. tecunumanii used in the study.

\begin{tabular}{|c|c|c|c|c|c|c|c|c|}
\hline Provenance & Code & $\begin{array}{l}\text { Elevation } \\
\text { group }\end{array}$ & Country & $\begin{array}{l}\text { Altitude } \\
\text { (III) }\end{array}$ & $\begin{array}{l}\text { Latitude } \\
{ }^{\circ} \mathbf{N}\end{array}$ & $\begin{array}{l}\text { Longitude } \\
{ }^{\circ} \mathbf{W}\end{array}$ & $\begin{array}{l}\text { Rainfall } \\
\text { (mm) }\end{array}$ & $\begin{array}{l}\text { Temperature } \\
\left({ }^{\circ} \mathrm{C}\right)\end{array}$ \\
\hline Culmí & Cul & LE & Honduras & $550-650$ & $15^{\circ} 06^{\prime}$ & $85^{\circ} 21^{\prime}$ & 1325 & 24.3 \\
\hline Mt. Pine Ridge & $\mathrm{Mpr}$ & LE & Belize & $700-720$ & $17^{\circ} 00^{\prime}$ & $88^{\circ} 55^{\prime}$ & 1558 & 23.9 \\
\hline San Esteban & Est & LE & Honduras & $700-800$ & $15^{\circ} 22^{\prime}$ & $85^{\circ} 35^{\prime}$ & 1400 & 25.0 \\
\hline Jocón & Joc & LE & Honduras & $850-1100$ & $15^{\circ} 16^{1}$ & $86^{\circ} 55^{\prime}$ & 1400 & 21.7 \\
\hline Cusuco & Cus & $\mathrm{HF}$ & Honduras & $1500-1650$ & $15^{\circ} 30^{\prime}$ & $88^{\circ} 10^{\prime}$ & 2500 & 17.0 \\
\hline La Paz & $\mathrm{Paz}$ & $\mathrm{HE}$ & Honduras & $1750-2000$ & $14^{\circ} 19^{\prime}$ & $87^{\circ} 45^{\prime}$ & 1619 & 20.0 \\
\hline Guajiquiro & Gua & $\mathrm{HE}$ & Honduras & $1835-2250$ & $14^{\circ} 11^{\prime}$ & $87^{\circ} 50^{\prime}$ & 2000 & 15.0 \\
\hline San Jerónimo & Jer & $\mathrm{HE}$ & Guatemala & $1700-2000$ & $15^{\circ} 03^{\prime}$ & $90^{\circ} 18^{\prime}$ & 1600 & 17.0 \\
\hline Montana Sumpu] & Sum & $\mathrm{HE}$ & Honduras & $1950-2050$ & $14^{\circ} 24^{\prime}$ & $89^{\circ} 08^{1}$ & 2200 & 16.0 \\
\hline
\end{tabular}

HE, LE - High Elevation and Low Elevation P. tecucunumanii.

2000a). It has been described as a magnificent tree, which usually forms mixed or pure stands of great commercial value in its natural range (EGUILUZ, 1986). Mature naturally-occurring stands of $P$. tecunumanii possess phenotypes with straight, clear boles and light narrow crowns. Twenty years ago, specimens that reach over $50 \mathrm{~m}$ in height and over $1 \mathrm{~m}$ diameter at breast height were not uncommon on good sites making this one of the largest of any of the tropical pines (STYLES and MCCARTER, 1988). However, many of the old growth forests have now been harvested by wood cutters.

The species has been separated into two subpopulations referred to as high and low elevation $P$. tecunumanii, based on the altitude of their occurrence in natural stands as well as subtle morphological differences in bark and cone characteristics (DVORAK, 1986; DvORAK et al., 2000a). Subsequent molecular marker analysis using RAPD (FURMAN et al., 1997; FURMAN and DvORAK, 2005) and microsatellites (DvORAK et al., 2009) has confirmed that genetic differences between the two groups are apparent. Disease screening of seedlings of $P$. tecunumanii from $\mathrm{HE}$ and $\mathrm{LE}$ for Pitch canker (Fusarium circinatum) resistance has also shown significant differences (HoDGE and DvORAK, 2007). High elevation (HE) populations generally occur above $1500 \mathrm{~m}$ a.s.l and low elevation (LE) populations occur below 1500 m.a.s.l. In the natural range, trees of the HE sources reach heights of up to $55 \mathrm{~m}$, while trees of the LE sources only reach heights of about $30 \mathrm{~m}$.

The first reports of growth performance of $P$. tecunumanii in the tropics were those of provenances of the LE $P$. tecunumanii, which were originally introduced and evaluated as $P$. oocarpa. Subsequent tests included seed sources of both $\mathrm{HE}$ and LE $P$. tecunumanii. Most of these sources were found to outperform provenances of true $P$. oocarpa in volume production (CROCKFORD et al., 1988; BIRKS and BARNES, 1990; CROCKFORD et al., 1990; NYOKA and BARNES, 1995). In other studies elsewhere, DvorAK and SHAW (1992) found that HE P. tecunumanii families were more productive than commercial seedlots of $P$. patula and $P$. oocarpa by as much as 20 and 40 percent respectively in Brazil, Colombia and South Africa.
More recently, Moura and Dvorak (1998) also found that HE Central American provenances were more productive than HE Mexican sources in Brazil.

The Zimbabwe Forest Research Centre established four trials of $P$. tecunumanii across four diverse sites in the $1990 \mathrm{~s}$. What makes these trials unique is that they included good representation of both $\mathrm{HE}$ and LE populations across the entire natural range of $P$. tecunumanii on the same site. Furthermore, the inclusion of two commercially grown species, $P$. patula and $P$. oocarpa as controls also provided an opportunity to compare the growth performance of the LE and HE P. tecunumanii provenances against these widely planted species.

The objectives of this study were to use field trials in Zimbabwe to: (a) compare the productivity of the LE and HE $P$. tecunumanii for which differentiation was originally hypothesised based on subtle morphological differences (DVORAK, 1986), monoterpene composition (SQUillace and PERRY, 1992) and unique RAPD (GRATTAPAGLIA et al., 1993; FURMAN et al., 1997) and microsatellite markers (DvorAK et al., 2009); (b) contrast the performance of the LE $P$. tecunumanii Belize provenance against the other LE $P$. tecunumanii; (c) contrast the performance of the isolated HE $P$. tecunumanii Mexican provenances against the other HE $P$. tecunumanii; and to (d) compare the productivity of $P$. tecunumanii with that of $P$. patula and $P$. oocarpa in respectively high and low altitude environments.

In this paper, the Juquila source is referred to as $P$. tecunumanii which is how it was originally described in the 1990s when the field trials were established. Since then, it has been shown not to carry markers for $P$. tecunumanii and has been reclassified as atypical P. herrerae/P. pringlei (DvORAK et al., 2001; DvORAK, 2008).

\section{Materials and Methods}

Open-pollinated seeds were collected by the Oxford Forestry Institute from 160 mother trees representing 16 provenances of $P$. tecunumanii in its natural range. The mother trees were selected on the basis of pheno- 
Table 2. - Details of climatic and geographic factors of the four test sites.

\begin{tabular}{|c|c|c|c|c|c|c|c|}
\hline \multirow[t]{3}{*}{ Site } & \multirow{2}{*}{$\begin{array}{l}\text { Altitude } \\
\text { a.s.l. }\end{array}$} & \multirow[t]{2}{*}{ Latitude } & \multirow[t]{2}{*}{ Longitude } & \multirow{2}{*}{$\begin{array}{l}\text { Rainfall } \\
\text { Annual }\end{array}$} & \multicolumn{3}{|c|}{ Mean Temperature ${ }^{\circ} \mathrm{C}$} \\
\hline & & & & & Annual & Maximum & Minimum \\
\hline & (m) & ${ }^{\circ} \mathrm{S}$ & ${ }^{\circ} \mathrm{E}$ & (mm) & $\left({ }^{\circ} \mathrm{C}\right)$ & $\left({ }^{\circ} \mathrm{C}\right)$ & $\left({ }^{\circ} \mathrm{C}\right)$ \\
\hline Stapleford & 1760 & $18^{\circ} 41$ & $32^{\circ} 51$ & 2159 & 15.1 & 19.2 & 11.1 \\
\hline Gungunyana & 1050 & $20^{\circ} 24$ & $32^{\circ} 43$ & 1097 & 18.1 & 24.0 & 13.9 \\
\hline Cashel & 1450 & $19^{\circ} 25$ & $32^{\circ} 45$ & 891 & 19.5 & 24.4 & 14.6 \\
\hline Maswera & 780 & $18^{\circ} 41$ & $32^{\circ} 55$ & 1498 & 20.7 & 28.4 & 13.0 \\
\hline
\end{tabular}

typic superiority in branch quality, stem form, tree size and taper. The selected mother trees in the wild were separated by a minimum distance of $100 \mathrm{~m}$ to avoid possibility of excessive neighbourhood inbreeding. Eight provenances were from altitudes above $1500 \mathrm{~m}$ a.s.l. (HE P. tecunumanii) and the other eight where from below this elevation (LE $P$. tecunumanii). Ten mother trees were selected in each provenance making a total of 80 families each for the LE and HE P. tecunumanii. Table 1 shows summaries of geographic location and climatic data of the provenances. One of the eight LE $P$. tecunumanii provenances (Mt Pine Ridge) is from Belize, while two of the eight HE $P$. tecunumanii are from Mexico (Las Piedrecitas and Juquila).

For the controls, $P$. patula seed was collected from 10 second generation clones in a clonal seed orchard that was available for commercial planting while seed for $P$. oocarpa was collected from 10 first generation selection clones in clonal seed orchards that were also currently being planted. The seed was sown at the Mukandi nursery near John Meikle Forest Research Station, Penhalonga (altitude $1246 \mathrm{~m}$ a.s.l.; rainfall $1778 \mathrm{~mm}$; temperature $18^{\circ} \mathrm{C}$ ). Sufficient seedlings were raised to establish orthogonal trials at four sites, ranging in altitude from 780 to $1760 \mathrm{~m}$ a.s.l. in the pine plantation growing areas of the eastern border of Zimbabwe. The details of geographic location and climatic factors of the test sites are shown in Table 2.

Stapleford is considered a favourable environment because of the high rainfall, deep, fertile and well drained soils. Although, rainfall at this site is received in summer, significant amounts are also received during the winter months. Although Cashel receives less than $1000 \mathrm{~mm}$ per annum, the cooler temperatures, moderated by altitude improve the effectiveness of the rainfall, making it an above average site. Gungunyana is considered an average environment as the soils are shallow and less fertile and rainfall is only received in summer, and significant winter rains are infrequent. Despite Maswera being a fairly wet site, the high temperatures that promote excessive water loss by evaporation coupled with insignificant precipitation in winter make this environment the least favourable among the four sites.

\section{Trial Design}

The trial design was a split plot in a randomised complete block with five replications at each site. Two commercial species, $P$. patula and $P$. oocarpa each with 10 families were included as control checks. The 16 provenances and the 2 species controls were randomised in main plots and the 10 families of each provenance and species were in turn randomised in subplots of each of the main plots. Each family subplot was a five tree line with a spacing of $3 \mathrm{~m}$ between rows and $3 \mathrm{~m}$ within the row. The main plot was therefore a $10 \times 5$ rectangular tree plot. There were a total 18 main plot treatments (16 provenances and 2 species) and 180 family subplots. The trial design was balanced at establishment for all the sites. The four trials were assessed for survival (sur2) and height (hgt2) at two years; height (hgt5), diameter at breast height (dbh5) and stem straightness (str5) at five years; and height (hgt8), diameter at breast height (dbh8) and stem straightness (str8) at eight years. Stem straightness was assessed on a scale of 1 to 7 . A rating of 1 represented the most crooked stem and 7 represented a very straight stem (BARNES and GIBSON, 1986).

\section{Statistical Analysis}

Trees that had severe stem breakage were excluded from the analysis. These were trees that had abnormal height:dbh ratios. Stem breakage averaged less than 10 percent at the worst affected site. Individual tree volume $\left(\mathrm{dm}^{3}\right)$ over bark at ages five and eight years was calculated using a formula for juvenile pines (DVORAK and SHAW, 1992):

$$
\text { vol }=0.3 *(\mathrm{dbh}) 2 * \text { height }
$$

where

vol = volume in cubic decimetres $\left(\mathrm{dm}^{3}\right)$ at five or eight years

$\mathrm{dbh}=$ diameter at breast height $(1.3 \mathrm{~m})$ in $\mathrm{cm}$ at five or eight years

height $=$ Height in metres at five or eight years.

The statistical analysis was conducted using PROC GLM in Statistical Analysis System (SAS, 1998). All the analyses were based on family subplot means rather than individual trees. The first analysis was on the individual site data and the following model was fitted to test the significance of all the effects and the contrasts:

$$
y_{i j k}=\mu+b_{i}+p_{j}+b p_{i j}+f(p)_{j(k)}+\varepsilon_{i j k}
$$

where

$y_{i j k}=$ observation in the $\mathrm{ijk}^{\text {th }}$ plot

$\mu \quad=$ general trial mean

$b_{i} \quad=$ effect of the $\mathrm{i}^{\text {th }}$ block

$p_{j} \quad=$ effect of the $\mathrm{j}^{\text {th }}$ provenance

$b p_{i j}=$ interaction effect between the $\mathrm{i}^{\text {th }}$ block and the

$j^{\text {th }}$ provenance or main plot error

$f(p)_{j k}=$ effect of the $\mathrm{k}^{\text {th }}$ family of $\mathrm{j}^{\text {th }}$ provenance

$\varepsilon_{i j k}=$ plot error. 
The four orthogonal contrasts tested with the model were:

(a) LE P. tecunumanii vs HE P. tecunumanii

(b) LE P. tecunumanii vs LE Belize sources (Mt Pine Ridge)

(c) HE P. tecunumanii vs HE Mexican sources (Las Piedrecitas and Juquila)

(d) $P$. tecunumanii vs $P$. patula (at Stapleford and Cashel) or $P$. oocarpa (at Gungunyana and Maswera).

The data was further analysed across sites to test the significance of the interactions. Data from the two controls were not used in the across site analysis. The appropriate model for this analysis was:

$$
\begin{aligned}
y_{i j k l m}= & \mu+s_{i}+b(s)_{i j}+a_{k}+s a_{i k}+a b(s)_{i j k}+p(a)_{k l}+ \\
& s p(a) i k l+b p(s a)_{i j k l}+f(p a)_{k l m}+s f(p a)_{i k l m}+e_{i j k l m}
\end{aligned}
$$

where

$y_{i j k l m}=$ the observation in the ijklm ${ }^{\text {th }}$ family subplot

$\mu \quad=$ the general mean

$s_{i} \quad=$ the effect of the $\mathrm{i}^{\text {th }}$ site

$b(s)_{i j} \quad=$ the effect of the $\mathrm{j}^{\text {th }}$ block in the $\mathrm{i}^{\text {th }}$ site

$a_{k} \quad=$ the effect of the $\mathrm{k}^{\text {th }} P$. tecunumanii type

$s a_{i k} \quad=$ the interaction of $\mathrm{i}^{\text {th }}$ site and $\mathrm{k}^{\text {th }} P$. tecunumanii type

$a b(s)_{i j k}=$ the interaction of $\mathrm{k}^{\text {th }} P$. tecunumanii type and $\mathrm{j}^{\text {th }}$ block in the $\mathrm{i}^{\text {th }}$ site

$p(a)_{k l} \quad=$ the effect of the $1^{\text {th }}$ provenance within the $\mathrm{k}^{\text {th }} P$. tecunumanii type

$s p(a)_{i k l}=$ the interaction of the $\mathrm{i}^{\text {th }}$ site with the $\mathrm{l}^{\text {th }}$ provenance of the $\mathrm{k}^{\text {th }} P$. tecunumanii type

$b p(s a)_{i j k l}=$ the interaction of the $\mathrm{j}^{\text {th }}$ block of the $\mathrm{i}^{\text {th }}$ site with the $\mathrm{l}^{\text {th }}$ provenance within the $\mathrm{k}^{\text {th }}$ $P$. tecunumanii type

$f(p a)_{k l m}=$ the effect of the $\mathrm{m}^{\text {th }}$ family within the $\mathrm{l}^{\text {th }}$ provenance within the $\mathrm{k}^{\text {th }} P$. tecunumanii type $s f(p a)_{i k l m}=$ the interaction of the $\mathrm{m}^{\text {th }}$ family of the $\mathrm{l}^{\text {th }}$ provenance of the $\mathrm{k}^{\text {th }} P$. tecunumanii type with the $\mathrm{i}^{\text {th }}$ site

$e_{i j k l m}=$ plot error.

\section{Results}

Tree survival at two years was above $92 \%$ for both the LE and HE $P$. tecunumanii at Stapleford and Cashel, but was down to $89 \%$ and $83 \%$ respectively at Gungunyana and Maswera (Table 3). Four HE provenances namely Guajiquiro, San Jeronimo, La Soledad and Las Piedrecitas had survival rates below $80 \%$. These provenances contributed to the overall lower mean survival rate of the HE $P$. tecunumanii at Maswera.

Table 4 shows the variance ratios of different sources of variation together with the four contrasts for two-, five- and eight-year growth and stem straightness at individual sites. Provenance effects were significant $(\mathrm{P}<0.001)$ at all the four sites for the growth and stem straightness traits. The LE and HE $P$. tecunumanii were significantly different from each other at lower altitude test sites (Gungunyana and Maswera) for all of the traits except stem straightness at eight years but the differences were often non-significant for some of the traits at the higher altitude sites (Stapleford and Cashel). The two Mexican HE P. tecunumanii provenances (Juquila and Las Piedrecitas) were significantly different from the other HE P. tecunumanii for most of the traits. The differences were more apparent at Gungunyana and Maswera but less pronounced at higher altitude sites of Stapleford and Cashel. The contrast between the Mt Pine Ridge and other LE P. tecunumanii was non-significant at Maswera, but was however significant at the other three sites for some of the traits. Also, the contrasts ( $P$. patula versus $P$. tecunumanii) at Stapleford and Cashel and ( $P$. oocarpa versus $P$. tecunumanii) at Gungunyana and Maswera were significant at varying probability levels for most of the traits.

Table 3. - Mean percent survival of $P$. tecunumanii provenances at individual sites at age two years.

\begin{tabular}{|l|l|l|l|l|}
\hline \multirow{2}{*}{ Provenance } & \multicolumn{3}{|c|}{ Site } \\
\cline { 2 - 5 } & Stapleford & Cashel & Gungunyana & Maswera \\
\hline Yucul & $93.2 \mathrm{ab}$ & $94.0 \mathrm{a}$ & $93.2 \mathrm{abcd}$ & $86.4 \mathrm{bcd}$ \\
Culmi & $88.0 \mathrm{~b}$ & $92.0 \mathrm{a}$ & $84.0 \mathrm{ef}$ & $80.4 \mathrm{de}$ \\
Villa Santa & $96.0 \mathrm{ab}$ & $90.0 \mathrm{a}$ & $89.2 \mathrm{bcde}$ & $86.4 \mathrm{bcd}$ \\
Mt Pine Ridge & $98.0 \mathrm{a}$ & $95.2 \mathrm{a}$ & $87.6 \mathrm{cde}$ & $92.8 \mathrm{abc}$ \\
Estaban & $87.6 \mathrm{~b}$ & $90.4 \mathrm{a}$ & $94.0 \mathrm{abc}$ & $89.2 \mathrm{abcd}$ \\
Jocón & $91.6 \mathrm{ab}$ & $94.4 \mathrm{a}$ & $95.6 \mathrm{ab}$ & $89.2 \mathrm{abcd}$ \\
San Francisco & $92.4 \mathrm{ab}$ & $95.2 \mathrm{a}$ & $94.8 \mathrm{ab}$ & $96.8 \mathrm{a}$ \\
Las Victorias & $92.0 \mathrm{ab}$ & $92.8 \mathrm{a}$ & $88.0 \mathrm{cde}$ & $87.2 \mathrm{abcd}$ \\
Cusuco & $89.6 \mathrm{ab}$ & $92.8 \mathrm{a}$ & $96.8 \mathrm{a}$ & $86.0 \mathrm{bcd}$ \\
La Paz & $93.6 \mathrm{ab}$ & $94.8 \mathrm{a}$ & $94.0 \mathrm{abc}$ & $85.6 \mathrm{bcd}$ \\
Guajiquiro & $88.0 \mathrm{~b}$ & $91.2 \mathrm{a}$ & $84.8 \mathrm{ef}$ & $79.2 \mathrm{de}$ \\
Jerónimo & $94.4 \mathrm{ab}$ & $96.0 \mathrm{a}$ & $85.2 \mathrm{ef}$ & $79.2 \mathrm{de}$ \\
Montana Sumpul & $96.8 \mathrm{ab}$ & $92.4 \mathrm{a}$ & $97.2 \mathrm{a}$ & $93.2 \mathrm{ab}$ \\
Juquila & $94.8 \mathrm{ab}$ & $96.8 \mathrm{a}$ & $86.8 \mathrm{def}$ & $82.8 \mathrm{cde}$ \\
La Soledad & $88.8 \mathrm{ab}$ & $92.4 \mathrm{a}$ & $80.4 \mathrm{fg}$ & $80.0 \mathrm{de}$ \\
Las Piedrecitas & $96.4 \mathrm{ab}$ & $94.4 \mathrm{a}$ & $74.4 \mathrm{~g}$ & $75.2 \mathrm{e}$ \\
\hline LE P. tecunumanii & 92.4 & 93.0 & 90.8 & 88.6 \\
HE P. tecunumanii & 93.1 & 93.9 & 87.5 & 82.7
\end{tabular}

Means in a column followed a common letters do not differ significantly at $5 \%$. 
Table 4. - ANOVA F-values for two-, five- and eight-year height, diameter, volume and stem straightness at individual sites.

\begin{tabular}{|c|c|c|c|c|c|c|c|c|c|c|}
\hline Source of Variation & DF & hgt2 & hgt5 & dbh5 & vol5 & str5 & hgt8 & dbh8 & vol8 & str8 \\
\hline \multicolumn{11}{|l|}{ Stapleford } \\
\hline Block & 4 & $123.7^{* * *}$ & $67.36^{* * *}$ & $25.96^{* * *}$ & $58.76^{* * *}$ & $12.56^{* * *}$ & $60.50^{* * *}$ & $12.78^{* * *}$ & $39.12^{* * *}$ & $32.16^{* * *}$ \\
\hline Provenance & 17 & $4.69^{* * *}$ & $6.95^{* * *}$ & $5.48^{* * *}$ & $9.13^{* * *}$ & $15.29^{* * *}$ & $9.85^{* * *}$ & $3.09^{* * *}$ & $6.34^{* * *}$ & $20.41^{* * *}$ \\
\hline HE vs LE $P$. tecunumanii & 1 & $33.87^{* * *}$ & $3.21^{\mathrm{ns}}$ & $0.84^{\mathrm{ns}}$ & $7.22^{\mathrm{ns}}$ & $73.28^{* * *}$ & $9.54^{* *}$ & $1.52^{\mathrm{ns}}$ & $7.69^{* *}$ & $108.5^{* * *}$ \\
\hline Mexico vs HE $P$. tecunumanii & 1 & $4.12^{a}$ & $0.29^{\mathrm{ns}}$ & $1.72^{\mathrm{ns}}$ & $0.79^{\mathrm{ns}}$ & $10.90^{* *}$ & $4.68^{*}$ & $4.53^{*}$ & $4.83^{*}$ & $23.20^{* * *}$ \\
\hline Belize vs LE $P$. tecunumanii & 1 & $7.87^{* *}$ & $0.03^{\text {ns }}$ & $2.02^{\mathrm{ns}}$ & $2.07^{\mathrm{ss}}$ & $8.25^{* *}$ & $5.35^{*}$ & $6.14^{*}$ & $8.27^{* *}$ & $14.40^{* * *}$ \\
\hline$P$. tecunumanii vs $P$. patula & 1 & $0.06^{\mathrm{ns}}$ & $42.65^{* * *}$ & $40.14^{* * *}$ & $78.45^{* * *}$ & $104.7^{* * *}$ & $54.75^{* * *}$ & $11.75^{* * *}$ & $32.22^{* * *}$ & $126.2^{* * *}$ \\
\hline Block*Provenance & 68 & & & & & & & & & \\
\hline $\begin{array}{l}\text { Family(Provenance) } \\
\text { Plot error }\end{array}$ & $\begin{array}{l}161 \\
649\end{array}$ & $2.38^{* * * *}$ & $1.73^{* * * *}$ & $1.53^{* * * *}$ & $1.71^{* * *}$ & $1.19^{\mathrm{ns}}$ & $1.76^{* * * *}$ & $1.59^{* * *}$ & $1.94^{* * *}$ & $2.14^{* * *}$ \\
\hline \multicolumn{11}{|l|}{ Cashel } \\
\hline Block & 4 & $21.87^{* * *}$ & $19.69^{* * *}$ & $27.92^{* * *}$ & $23.97^{* * *}$ & $5.23^{* * *}$ & $4.69^{* *}$ & $12.91^{* * *}$ & $11.67^{* * *}$ & $8.65^{* * *}$ \\
\hline Provenance & 17 & $7.47^{*+*}$ & $5.53^{* * *}$ & $5.34^{* * *}$ & $7.58^{* * *}$ & $18.92^{* * *}$ & $5.06^{* * *}$ & $3.42^{* * *}$ & $4.67^{*+*}$ & $20.11^{* * *}$ \\
\hline HE vs LE $P$. tecunumanii & 1 & $41.13^{* * *}$ & $28.93^{* * *}$ & $25.95^{* * *}$ & $37.83^{* * *}$ & $76.80^{* * *}$ & $3.49^{\mathrm{ns}}$ & $0.85^{\mathrm{ns}}$ & $0.06^{\mathrm{ns}}$ & $121.6^{* * *}$ \\
\hline Mexico vs HE $P$. tecunumanii & 1 & $4.69^{*}$ & $3.93^{\text {ns }}$ & $0.40^{\text {ns }}$ & $1.36^{\mathrm{ns}}$ & $45.99^{* * *}$ & $1.08^{\mathrm{ns}}$ & $1.59^{\mathrm{ns}}$ & $0.21^{\mathrm{ns}}$ & $32.28^{* * *}$ \\
\hline Belize vs Low $P$. tecunumanii & 1 & $1.78^{\mathrm{ns}}$ & $0.08^{\mathrm{ns}}$ & $2.52^{\mathrm{ns}}$ & $4.45^{*}$ & $9.02^{* *}$ & $15.51^{* * *}$ & $8.84^{* *}$ & $14.15^{* * *}$ & $14.28^{* * *}$ \\
\hline$P$. tecunumanii vs $P$. patula & 1 & $27.53^{* * *}$ & $7.16^{* *}$ & $17.34^{* * *}$ & $14.61^{* * *}$ & $120.0^{* * *}$ & $3.76^{\mathrm{ns}}$ & $0.52^{\mathrm{ns}}$ & $0.06^{\mathrm{ns}}$ & $141.1^{* * *}$ \\
\hline Block*Provenance & 68 & & & & & & & & & \\
\hline $\begin{array}{l}\text { Family(Provenance) } \\
\text { Plot error }\end{array}$ & $\begin{array}{l}161 \\
649\end{array}$ & $3.76^{* * *}$ & $2.73^{* * *}$ & $2.42^{* * *}$ & $2.32^{* * *}$ & $1.75^{* * *}$ & $2.51^{*}$ & $2.54^{* * *}$ & $2.17^{* * *}$ & $1.95^{* * *}$ \\
\hline \multicolumn{11}{|l|}{ Gungunyana } \\
\hline Block & 4 & $8.12^{* * *}$ & $8.61^{* * *}$ & $11.05^{* * *}$ & $15.98^{* * *}$ & $2.21^{\mathrm{ns}}$ & $8.02^{* * *}$ & $9.03^{* * *}$ & $12.17^{* * *}$ & $17.07^{* * *}$ \\
\hline Provenance & 17 & $18.41^{* * *}$ & $17.98^{* * *}$ & $11.41^{* * *}$ & $15.20^{* * *}$ & $4.67^{* * *}$ & $12.13^{* * *}$ & $2.50^{* *}$ & $5.02^{* * *}$ & $7.24^{* * *}$ \\
\hline HE vs LE $P$. tecunumanii & 1 & $81.17^{* * *}$ & $135.4^{* * *}$ & $65.11^{* * *}$ & $82.62^{* * *}$ & $11.54^{* *+}$ & $85.22^{* * *}$ & $19.33^{* * *}$ & $36.50^{* * *}$ & $9.44^{* *}$ \\
\hline Mexico vs HE $P$. tecunumanii & 1 & $12.75^{* * *}$ & $54.28^{* * *}$ & $25.97^{* * *}$ & $27.57^{* * *}$ & $0.36^{\mathrm{ns}}$ & $48.09^{* * *}$ & $3.99^{*}$ & $12.86^{* * *}$ & $2.50^{\mathrm{ns}}$ \\
\hline Belize vs LE $P$. tecunumanii & 1 & $6.87^{*}$ & $16.04^{* * *}$ & $10.67^{* *}$ & $15.16^{* * *}$ & $6.68^{*}$ & $38.93^{* * *}$ & $7.77^{* *}$ & $15.10^{* * *}$ & $24.49^{* * *}$ \\
\hline$P$. tecunumanii vs $P$. oocarpa & 1 & $28.00^{* * *}$ & $1.01^{\mathrm{ns}}$ & $6.43^{*}$ & $7.35^{* *}$ & $1.95^{\mathrm{ns}}$ & $5.60^{*}$ & $0.23^{\text {ns }}$ & $1.38^{\mathrm{ns}}$ & $1.63^{\mathrm{ns}}$ \\
\hline Block*Provenance & 68 & & & & & & & & & \\
\hline $\begin{array}{l}\text { Family (Provenance) } \\
\text { Plot error }\end{array}$ & $\begin{array}{l}161 \\
649\end{array}$ & $2.18^{* * *}$ & $2.41^{* * *}$ & $2.19^{* * *}$ & $2.17^{* * *}$ & $1.72^{* *}$ & $2.49^{* * *}$ & $1.91^{* * *}$ & $2.05^{* * *}$ & $1.79^{* * *}$ \\
\hline \multicolumn{11}{|l|}{ Maswera } \\
\hline Block & 4 & $47.31^{* * *}$ & $146.1^{* * *}$ & $132.1^{* * *}$ & $121.1^{* * *}$ & $84.63^{* * *}$ & $86.57^{* * *}$ & $55.15^{* * *}$ & $66.73^{* * *}$ & $20.08^{* * *}$ \\
\hline Provenance & 17 & $19.59^{* * *}$ & $19.67^{* * *}$ & $19.79^{* * *}$ & $18.13^{* * *}$ & $8.78^{* * * *}$ & $17.86^{* * *}$ & $15.65^{* * *}$ & $14.73^{* * *}$ & $3.07^{* * *}$ \\
\hline HE vs LE $P$. tecunumanii & 1 & $132.4^{* * *}$ & $120.4^{* * *}$ & $81.28^{* * *}$ & $84.38^{* * *}$ & $29.90^{* * *}$ & $91.75^{* * *}$ & $68.69^{* * *}$ & $75.85^{* * *}$ & $0.20^{\mathrm{ns}}$ \\
\hline Mexico vs HE $P$. tecunumanii & 1 & $25.37^{* * *}$ & $40.78^{* * *}$ & $30.78^{* * *}$ & $23.70^{* * *}$ & $5.00^{*}$ & $43.81^{* * *}$ & $18.93^{* * *}$ & $23.94^{* * *}$ & $1.34^{\mathrm{ns}}$ \\
\hline Belize vs LE $P$. tecunumanii & 1 & $3.02^{\mathrm{ns}}$ & $0.78^{\text {ns }}$ & $0.00^{\mathrm{ns}}$ & $2.13^{\text {ns }}$ & $1.47^{\mathrm{ns}}$ & $0.20^{\text {ns }}$ & $2.33^{\text {ns }}$ & $0.00^{\mathrm{ns}}$ & $6.67^{*}$ \\
\hline$P$. tecunumanii vs $P$. patula & 1 & $11.40^{* *}$ & $9.12^{* *}$ & $26.30^{* * *}$ & $21.15^{* * *}$ & $20.69^{* * *}$ & $7.82^{* *}$ & $16.17^{* * *}$ & $11.77^{* * *}$ & $2.86^{\mathrm{ns}}$ \\
\hline Block*Provenance & 68 & & & & & & & & & \\
\hline Family(Provenance) & 161 & $3.15^{* * *}$ & $2.41^{* * *}$ & $2.16^{* * *}$ & $2.09^{* * *}$ & $1.58^{* *}$ & $2.06^{* * *}$ & $1.85^{* *}$ & $1.91^{* *}$ & $1.44^{* *}$ \\
\hline Plot error & 649 & & & & & & & & & \\
\hline
\end{tabular}

ns, a, b, c for not significant at $5 \%$, significant at 5,1 and $0.1 \%$ respectively.

Belize refers to the Mt Pine Ridge provenance.

Mexico refers to the Las Piedrecitas and Juquila provenance.

Tables 5 to 8 show the two-, five- and eight-year provenance means of growth and stem straightness together with the means of the LE and HE $P$. tecunumanii at individual sites. Family ranges in these same traits are shown in Table 9. Provenance rank changes in height, diameter and volume growth with age was evident at all the four sites, with provenances from the LE $P$. tecunumanii showing exceptional growth in the early years. The most notable provenance for changing rank with age was Mt Pine Ridge, which was among the top

Table 5. - Means of growth and stem straightness of P. tecunumanii provenances at Stapleford.

\begin{tabular}{|c|c|c|c|c|c|c|c|c|c|c|c|c|c|c|c|c|c|}
\hline \multirow[t]{2}{*}{ Provenance } & \multicolumn{17}{|c|}{ Means } \\
\hline & $\begin{array}{l}\text { vol } 8 \\
\left(\mathrm{dm}^{3}\right)\end{array}$ & $\begin{array}{l}\mathrm{dbh} 8 \\
(\mathrm{~cm})\end{array}$ & rk & $\begin{array}{l}\text { hgt } 8 \\
\text { (m) }\end{array}$ & rk & $\begin{array}{l}\text { str8 } \\
(1-7)\end{array}$ & rk & $\begin{array}{l}\text { vol5 } \\
\left(\mathrm{dm}^{3}\right) \\
\end{array}$ & rk & $\begin{array}{l}\text { dbh5 } \\
(\mathrm{cm})\end{array}$ & rk & $\begin{array}{l}\text { hgt5 } \\
\text { (m) }\end{array}$ & rk & $\begin{array}{l}\text { str5 } \\
(1-7)\end{array}$ & $\mathrm{rk}$ & $\begin{array}{l}\text { hgt2 } \\
(\mathrm{m})\end{array}$ & $\mathrm{rk}$ \\
\hline Juquila. & $2089.2 a$ & $20.2 \mathrm{a}$ & 1 & $13.5 \mathrm{a}$ & 1 & $4.2 \mathrm{a}$ & 1 & $486.2 \mathrm{ab}$ & 2 & $12.6 a$ & I & $7.8 \mathrm{a}$ & 4 & $3.8 \mathrm{a}$ & l & $1.2 \mathrm{ab}$ & 13 \\
\hline Guajiquiro & $1861.0 \mathrm{ab}$ & $19.5 \mathrm{ab}$ & 2 & $13.0 \mathrm{ab}$ & 3 & $3.7 \mathrm{bcde}$ & 5 & $440.9 \mathrm{abc}$ & 5 & $11.9 \mathrm{a}$ & 6 & $7.9 \mathrm{a}$ & 3 & $3.6 a b c$ & 4 & $1.2 \mathrm{ab}$ & 13 \\
\hline La Soledad & 1791.3abc & $19.0 \mathrm{abcd}$ & 5 & $12.7 \mathrm{ab}$ & 7 & $3.5 \mathrm{dcfg}$ & 9 & $432.5 \mathrm{abcd}$ & 6 & $11.9 \mathrm{a}$ & 6 & $7.4 a b$ & 10 & 3.5 abcd & 5 & $1.1 \mathrm{~b}$ & 16 \\
\hline Villa Santa. & $1788.0 \mathrm{abc}$ & $19.3 \mathrm{abc}$ & 3 & $12.7 \mathrm{ab}$ & 7 & 3.5 defg & 9 & 468.0abed & 3 & $12.5 \mathrm{a}$ & 2 & $7.7 \mathrm{ab}$ & 5 & 3.3bode & 9 & $1.5 \mathrm{a}$ & 1 \\
\hline San Jerónimo & $1784.3 \mathrm{abc}$ & $18.6 \mathrm{abcd}$ & 8 & $13.5 \mathrm{a}$ & 1 & $4.1 \mathrm{ab}$ & 2 & $495.7 \mathrm{a}$ & 1 & $12.5 \mathrm{a}$ & 2 & $8.2 \mathrm{a}$ & 1 & $3.7 \mathrm{ab}$ & 2 & $1.3 \mathrm{ab}$ & 7 \\
\hline Yucul & $1740.7 \mathrm{cbc}$ & 19.labcd & 4 & $12.7 \mathrm{ab}$ & 7 & 3.3 efgh & 11 & $405.5 \mathrm{abcd}$ & 9 & $11.7 \mathrm{a}$ & 8 & $7.7 \mathrm{ab}$ & 5 & 3.3 bcde & 9 & $1.3 \mathrm{ab}$ & 7 \\
\hline San Estaban & $1645.2 \mathrm{bed}$ & 19.0abed & 5 & $12.0 \mathrm{~b}$ & 14 & $3.0 \mathrm{~h}$ & 16 & $405.7 \mathrm{abcd}$ & 8 & $12.0 \mathrm{a}$ & 5 & $7.2 \mathrm{ab}$ & 13 & $3.1 \mathrm{de}$ & 15 & $1.4 \mathrm{ab}$ & 3 \\
\hline Jocón & $1638.8 \mathrm{bcd}$ & 18.6abcd & 8 & $12.2 \mathrm{ab}$ & 12 & $3.2 \mathrm{fgh}$ & 13 & 386.4abed & 11 & $11.5 \mathrm{a}$ & 10 & $7.2 \mathrm{ab}$ & 13 & $3.2 \mathrm{cde}$ & 12 & $1.4 \mathrm{ab}$ & 3 \\
\hline Las Piedrecitas & $1637.5 \mathrm{bcd}$ & 18.4abcd & 10 & $12.8 \mathrm{ab}$ & 5 & 4.0abc & 3 & $387.3 \mathrm{abcd}$ & 12 & $11.4 \mathrm{a}$ & 12 & $7.6 \mathrm{ab}$ & 8 & 3.5abcd & 5 & $1.2 \mathrm{ab}$ & 13 \\
\hline San Francisco & $1587.4 \mathrm{bcd}$ & $18.1 \mathrm{bcd}$ & 12 & $12.8 \mathrm{ab}$ & 5 & $3.6 \mathrm{cdcf}$ & 6 & $330.5 d$ & 16 & $11.1 \mathrm{a}$ & 13 & $6.7 \mathrm{~b}$ & 16 & $3.3 \mathrm{bcdc}$ & 9 & $1.4 a b$ & 3 \\
\hline Montana Sumpul & $1581.3 \mathrm{bcd}$ & 18.1bed & 12 & $12.6 \mathrm{ab}$ & 10 & $3.9 \mathrm{abcd}$ & 4 & $412.0 \mathrm{abcd}$ & 7 & $11.7 \mathrm{a}$ & 8 & $7.7 \mathrm{ab}$ & 5 & $3.7 \mathrm{ab}$ & 2 & $1.3 \mathrm{ab}$ & 7 \\
\hline Cusuco & $1580.0 \mathrm{bed}$ & $18.7 \mathrm{abcd}$ & 7 & $11.8 \mathrm{~b}$ & 15 & 3.3efgh & 11 & 391.2abcd & 10 & $11.5 \mathrm{a}$ & 10 & $7.3 a b$ & 11 & $3.2 \mathrm{cde}$ & 12 & $1.4 a b$ & 3 \\
\hline Las Victorias & $1573.5 \mathrm{bcd}$ & $18.2 \mathrm{bcd}$ & 11 & $12.9 \mathrm{ab}$ & 4 & $3.6 \mathrm{cdef}$ & 6 & $447.2 \mathrm{abcd}$ & 4 & $12.2 \mathrm{a}$ & 4 & 8.la & 2 & $3.5 \mathrm{abcd}$ & 5 & $1.3 \mathrm{ab}$ & 7 \\
\hline La.Paz & $1551.5 \mathrm{~cd}$ & $17.8 \mathrm{bed}$ & 15 & $12.4 \mathrm{ab}$ & 11 & $3.6 \mathrm{cdcf}$ & 6 & $373.3 \mathrm{bcd}$ & 13 & $11.0 \mathrm{a}$ & 16 & $7.2 \mathrm{ab}$ & 13 & 3.4abcde & 8 & $1.3 \mathrm{ab}$ & 7 \\
\hline Culmi & $1463.5 \mathrm{~cd}$ & $17.9 \mathrm{bed}$ & 14 & $12.2 \mathrm{ab}$ & 12 & 3.lgh & 14 & $355.7 \mathrm{bcd}$ & 14 & $11.1 \mathrm{~b}$ & 13 & $7.5 \mathrm{ab}$ & 9 & $3.2 \mathrm{cde}$ & 12 & $1.3 \mathrm{ab}$ & 7 \\
\hline Mt Pine Ridge & $1340.7 d$ & $17.3 \mathrm{~d}$ & 16 & $11.8 \mathrm{~b}$ & 15 & 3.lgh & 14 & $352.5 \mathrm{~cd}$ & 15 & $11.1 \mathrm{~b}$ & 13 & $7.3 \mathrm{ab}$ & 11 & $3.1 \mathrm{e}$ & 15 & $1.5 \mathrm{a}$ & 1 \\
\hline Low Elevation & 1588.6 & 18.4 & & 12.4 & & 3.5 & & 393.4 & & 11.6 & & 7.4 & & 3.2 & & 1.4 & \\
\hline High Elcvation & 1729.2 & 18.7 & & 12.8 & & 4.0 & & 428.1 & & 11.8 & & 7.6 & & 3.6 & & 1,2 & \\
\hline
\end{tabular}

Means in a column followed by a common letter do not differ significantly at $5 \%$; rk $=$ rank.

Low Elevation and High Elevation = Low and High Elevation P. tecunumanii. 
Table 6. - Means of growth and stem straightness of P. tecunumanii provenances at Cashel.

\begin{tabular}{|c|c|c|c|c|c|c|c|c|c|c|c|c|c|c|c|c|c|}
\hline \multirow[t]{2}{*}{ Provenance } & \multicolumn{17}{|c|}{ Means } \\
\hline & $\begin{array}{l}\text { vol8 } \\
\left(\mathrm{dm}^{3}\right) \\
\end{array}$ & $\begin{array}{l}\mathrm{dbh} 8 \\
(\mathrm{~cm})\end{array}$ & rk & $\begin{array}{l}\text { hgt8 } \\
\text { (m) }\end{array}$ & $\mathrm{rk}$ & $\begin{array}{l}\text { str8 } \\
(1-7)\end{array}$ & rk & $\begin{array}{l}\text { vol5 } \\
\left(\mathrm{dm}^{3}\right)\end{array}$ & $\mathrm{rk}$ & $\begin{array}{l}\text { dbh5 } 5 \\
(\mathrm{~cm})\end{array}$ & rk & $\begin{array}{l}\text { hgt5 } \\
(\mathrm{m})\end{array}$ & rk & $\begin{array}{l}\text { str5 } \\
(1-7)\end{array}$ & $\mathrm{rk}$ & $\begin{array}{l}\text { hgt2 } \\
\text { (m) }\end{array}$ & rk \\
\hline Yucul & $1383.6 \mathrm{a}$ & $17.5 a$ & 1 & $11.9 a$ & 2 & $3.9 \mathrm{bcd}$ & 1 & $296.6 \mathrm{a}$ & 1 & $10.0 \mathrm{a}$ & 1 & $7.5 \mathrm{a}$ & 1 & $3.5 a b c$ & 5 & $1.3 a b c$ & 3 \\
\hline Las Victorias & $1365.6 \mathrm{a}$ & $17.2 \mathrm{a}$ & 3 & $12.2 \mathrm{a}$ & 1 & $3.2 \mathrm{bcd}$ & 9 & $269.5 \mathrm{ab}$ & 3 & $9.6 \mathrm{a}$ & 2 & $7.1 \mathrm{ab}$ & 2 & $3.5 \mathrm{abc}$ & 5 & $1.3 a b c$ & 3 \\
\hline Guajiquiro & $1298.4 \mathrm{a}$ & $17.3 \mathrm{a}$ & 2 & $11.3 \mathbf{a}$ & 6 & $3.4 a b c$ & 4 & $201.5 b c$ & 8 & $8.7 \mathrm{a}$ & 9 & $6.4 \mathrm{ab}$ & 10 & $3.8 \mathrm{ab}$ & 3 & $1.1 \mathrm{bc}$ & 11 \\
\hline San Jerónimo & $1226.0 \mathrm{ab}$ & $16.7 \mathrm{ab}$ & 5 & $11.3 \mathrm{a}$ & 6 & $3.4 a b c$ & 4 & 196.Sbc & 11 & $8.6 \mathrm{a}$ & 10 & $6.5 \mathrm{ab}$ & 6 & $3.7 \mathrm{abc}$ & 4 & $1.2 \mathrm{bc}$ & 8 \\
\hline Juquila & $1224.2 \mathrm{ab}$ & $16.9 \mathrm{ab}$ & 4 & $11.4 \mathrm{a}$ & 4 & $3.8 \mathrm{a}$ & 2 & $182.5 b c$ & 12 & $8.5 \mathrm{a}$ & 12 & $6.2 \mathrm{ab}$ & 13 & $4.0 \mathrm{a}$ & 1 & $1.1 \mathrm{bc}$ & 11 \\
\hline San Francisco & $1216.4 \mathrm{ab}$ & $16.5 \mathrm{ab}$ & 7 & $11.6 \mathrm{a}$ & 3 & $2.9 \mathrm{~cd}$ & 14 & $271.1 \mathrm{ab}$ & 2 & $9.5 \mathrm{a}$ & 3 & $6.8 \mathrm{ab}$ & 3 & $3.3 b c$ & 12 & $1.6 \mathrm{a}$ & 1 \\
\hline Villa Santa & $1214.8 \mathrm{ab}$ & $16.7 \mathrm{ab}$ & 5 & $11.3 \mathrm{a}$ & 6 & $3.0 \mathrm{bcd}$ & 11 & $234.5 \mathrm{abc}$ & 4 & $9.4 \mathrm{a}$ & 4 & $6.5 \mathrm{ab}$ & 6 & $3.3 \mathrm{bc}$ & 12 & $1.2 b c$ & 8 \\
\hline Las Piedrecitas & $1161.6 \mathrm{ab}$ & $16.4 a b$ & 8 & $10.8 \mathrm{a}$ & 14 & $3.5 \mathrm{ab}$ & 3 & $166.5 \mathrm{c}$ & 16 & $8.0 \mathrm{a}$ & 15 & $5.9 \mathrm{~b}$ & 15 & $3.9 \mathrm{ab}$ & 2 & $1.0 \mathrm{c}$ & 15 \\
\hline La Paz & $1155.1 \mathrm{ab}$ & 16.1ab & 10 & $11.3 \mathrm{a}$ & 6 & $3.3 \mathrm{abc}$ & 6 & $207.3 b c$ & 7 & $8.8 a$ & 6 & $6.5 \mathrm{ab}$ & 6 & $3.6 \mathrm{abc}$ & 5 & $1.3 a b c$ & 3 \\
\hline La Soledad & $1147.3 \mathrm{ab}$ & $16.2 \mathrm{ab}$ & 9 & $11,3 \mathrm{a}$ & 6 & $3.3 \mathrm{abc}$ & 6 & $182.3 b c$ & 13 & $8.3 \mathrm{a}$ & 13 & $6.1 b$ & 14 & $3.6 \mathrm{abc}$ & 5 & $1.1 \mathrm{bc}$ & 11 \\
\hline Cusuco & $1116.3 \mathrm{ab}$ & $15.7 \mathrm{ab}$ & 13 & $11.1 \mathrm{a}$ & 11 & $3.2 \mathrm{bcd}$ & 9 & $209.0 \mathrm{bc}$ & 6 & $8.6 \mathrm{a}$ & 10 & $6.5 \mathrm{ab}$ & 6 & $3.3 \mathrm{bc}$ & 12 & $1.2 \mathrm{bc}$ & 8 \\
\hline San Estaban & $1113.5 \mathrm{ab}$ & $15.9 \mathrm{ab}$ & 11 & $11.4 \mathrm{a}$ & 4 & $3.0 \mathrm{bcd}$ & 11 & $219.3 \mathrm{abc}$ & 5 & $9.0 \mathrm{a}$ & 5 & $6.6 \mathrm{ab}$ & 4 & $3.3 \mathrm{bc}$ & 12 & $1.3 \mathrm{abc}$ & 3 \\
\hline Montana Sumpul & $1077.2 \mathrm{ab}$ & $15.9 \mathrm{ab}$ & 11 & $10.8 \mathrm{a}$ & 15 & $3.3 b c$ & 6 & $176.7 \mathrm{c}$ & 14 & $8.0 \mathrm{a}$ & 15 & $5.9 \mathrm{~b}$ & 15 & $3.5 \mathrm{abc}$ & 5 & $1.0 \mathrm{c}$ & 15 \\
\hline Jocón & $1055.6 \mathrm{ab}$ & $15.6 \mathrm{ab}$ & 14 & $11.1 \mathrm{a}$ & 11 & $3.0 \mathrm{bcd}$ & 11 & $200.9 \mathrm{bc}$ & 10 & $8.8 a$ & 6 & $6.4 a b$ & 10 & $3.5 \mathrm{abc}$ & 5 & $1.3 \mathrm{abc}$ & 3 \\
\hline Culmi & $953.9 \mathrm{~b}$ & $15.1 \mathrm{~b}$ & 15 & $11,0 \mathrm{a}$ & 13 & $2.9 \mathrm{~cd}$ & 14 & $166.6 \mathrm{c}$ & 15 & $8.1 \mathrm{a}$ & 14 & $6.3 \mathrm{ab}$ & 12 & $3.4 a b c$ & 11 & $1.1 b c$ & 11 \\
\hline Mt Pine Ridge & $927.1 \mathrm{~b}$ & $15.1 \mathrm{~b}$ & 15 & $10.6 \mathrm{a}$ & 16 & $2.7 \mathrm{~d}$ & 16 & $201.3 b c$ & 9 & $8.8 \mathrm{a}$ & 6 & $6.6 \mathrm{ab}$ & 4 & 3.lc & 16 & $1.4 \mathrm{ab}$ & 2 \\
\hline Low Elevation & 1143.9 & 16.2 & & 11.4 & & 3.0 & & 233.8 & & 9.2 & & 6.7 & & 3.4 & & 1.4 & \\
\hline Iligh Elevation & 1163.1 & 16.4 & & 11.2 & & 3.4 & & 191.4 & & 8.5 & & 6.3 & & 3.7 & & 1.2 & \\
\hline
\end{tabular}

Means in a column followed by a common letter do not differ significantly at $5 \%$; rk $=$ rank.

Low Elevation and High Elevation = Low and High Elevation P. tecunumanii.

Table 7. - Means of growth and stem straightness of $P$. tecunumanii provenances at Gungunyana.

\begin{tabular}{|c|c|c|c|c|c|c|c|c|c|c|c|c|c|c|c|c|c|}
\hline \multirow[t]{2}{*}{ Provenance } & \multicolumn{17}{|c|}{ Means } \\
\hline & $\begin{array}{l}\text { vol8 } \\
\left(\mathrm{dm}^{3}\right)\end{array}$ & $\begin{array}{l}\text { dbh8 } \\
\text { (cm) }\end{array}$ & rk & $\begin{array}{l}\text { hgt8 } \\
\text { (m) }\end{array}$ & $\mathrm{rk}$ & $\begin{array}{l}\text { str8 } \\
(1-7)\end{array}$ & $\mathrm{rk}$ & $\begin{array}{l}\text { vol5 } \\
\left(\mathrm{dm}^{3}\right)\end{array}$ & $\mathrm{rk}$ & $\begin{array}{l}\text { dbh5 } \\
\text { (cm) }\end{array}$ & rk & $\begin{array}{l}\text { hgt5 } \\
\text { (m) }\end{array}$ & $\mathrm{rk}$ & $\begin{array}{l}\text { str5 } \\
(1-7)\end{array}$ & $\mathrm{rk}$ & $\begin{array}{l}\text { hgt2 } \\
\text { (m) }\end{array}$ & $\mathrm{rk}$ \\
\hline S. Francisco & $1995.9 \mathrm{a}$ & $18.7 a$ & 1 & $15.1 \mathrm{ab}$ & 2 & $3.7 a b$ & 9 & $382.2 \mathrm{a}$ & 3 & $10.7 \mathrm{a}$ & 1 & $8.4 a$ & 1 & $3.4 a$ & 7 & $2.2 \mathrm{a}$ & 1 \\
\hline Yucul & $1958.7 \mathrm{ab}$ & $18.3 \mathrm{ab}$ & 2 & $15.4 \mathrm{a}$ & 1 & $3.9 \mathrm{ab}$ & 2 & $289.1 \mathrm{bc}$ & 2 & $9.6 \mathrm{ab}$ & 2 & $7.8 \mathrm{ab}$ & 2 & $3.5 \mathrm{a}$ & 4 & $1.5 \mathrm{bc}$ & 3 \\
\hline Las Victorias & $1794.3 \mathrm{abc}$ & $17.7 \mathrm{abcd}$ & 4 & $14.9 \mathrm{ab}$ & 3 & $3.8 \mathrm{abc}$ & 6 & $269,3 \mathrm{bcd}$ & 5 & $8.9 \mathrm{bcd}$ & 6 & $7.7 \mathrm{ab}$ & 3 & $3.5 \mathrm{a}$ & 4 & $1.5 \mathrm{bc}$ & 3 \\
\hline Villa Santa & $1791.1 \mathrm{abc}$ & $17.9 \mathrm{abc}$ & 3 & $14.5 \mathrm{abc}$ & 5 & $3.7 \mathrm{abc}$ & 9 & $226.4 \mathrm{def}$ & 8 & $8.7 \mathrm{bed}$ & 8 & $7.2 \mathrm{bc}$ & 6 & $3.3 \mathrm{a}$ & 15 & 1.3 code & 8 \\
\hline Cusuco & $1758.9 \mathrm{abc}$ & $17.3 \mathrm{abcd}$ & 8 & $14.1 \mathrm{abc}$ & 7 & $3.6 \mathrm{bc}$ & 14 & $297.2 \mathrm{~b}$ & 1 & $9.6 a b$ & 2 & $7.5 \mathrm{ab}$ & 5 & $3.4 a$ & 7 & $1.5 \mathrm{bc}$ & 3 \\
\hline San Estaban & $1745.2 \mathrm{abc}$ & $17.6 \mathrm{abcd}$ & 6 & $14.7 \mathrm{abc}$ & 4 & $3.7 \mathrm{abc}$ & 9 & $278.7 \mathrm{bc}$ & 4 & $9.5 \mathrm{abc}$ & 4 & $7.6 \mathrm{ab}$ & 4 & $3.4 \mathrm{a}$ & 7 & $1.6 b$ & 2 \\
\hline Culmi & $1700.8 \mathrm{bcd}$ & $17.7 \mathrm{abcd}$ & 4 & $14,2 \mathrm{abc}$ & 6 & $3.6 \mathrm{bc}$ & 14 & $222.8 \mathrm{def}$ & 9 & $8.7 \mathrm{bcd}$ & 8 & $7.2 b c$ & 6 & $3.4 \mathrm{a}$ & 7 & $1.3 \mathrm{cde}$ & 8 \\
\hline $\mathrm{LaPa}$ & $1649.8 \mathrm{cde}$ & 17.3abcd & 8 & $14.1 \mathrm{abc}$ & 7 & $3.9 \mathrm{ab}$ & 2 & $236.7 \mathrm{cde}$ & 7 & $8.9 \mathrm{bed}$ & 6 & $7.2 \mathrm{bc}$ & 6 & $3.4 \mathrm{a}$ & 7 & $1.3 \mathrm{cde}$ & 8 \\
\hline San Jcrónimo & $1633.3 \mathrm{cdc}$ & $17.5 \mathrm{abcd}$ & 7 & $14.0 \mathrm{abc}$ & 10 & $3.9 \mathrm{ab}$ & 2 & $185.7 \mathrm{cfg}$ & 11 & $8.2 \mathrm{cdcf}$ & 11 & $6.5 \mathrm{cde}$ & 11 & $3.6 \mathrm{a}$ & 1 & $1.2 \mathrm{dc}$ & 12 \\
\hline Jocón & $1623.1 \mathrm{cde}$ & 17.1 abed & 10 & $14,1 \mathrm{abc}$ & 7 & $3.7 \mathrm{abc}$ & 9 & $237.9 \mathrm{cde}$ & 6 & $9.3 \mathrm{bc}$ & 5 & $7.1 \mathrm{bc}$ & 9 & $3.4 \mathrm{a}$ & 7 & $1.5 \mathrm{bc}$ & 3 \\
\hline Mt Pine Ridge & $1457.8 \mathrm{def}$ & $17.1 \mathrm{bcd}$ & 10 & $13.2 \mathrm{~cd}$ & 13 & $3.4 \mathrm{c}$ & 16 & $206.8 \mathrm{ef}$ & 10 & $8.6 b c d$ & 10 & $7.0 \mathrm{bcd}$ & 10 & $3.2 \mathrm{a}$ & 16 & $1.4 \mathrm{bcd}$ & 7 \\
\hline Guajiquiro & $1455.9 \mathrm{def}$ & $16.6 \mathrm{bcd}$ & 12 & $13.3 \mathrm{~cd}$ & 12 & $3.8 \mathrm{abc}$ & 6 & $148.2 \mathrm{gh}$ & 14 & $7.3 \mathrm{c}$ & 14 & $6.1 \mathrm{def}$ & 14 & $3.6 \mathrm{a}$ & 1 & $1.1 \mathrm{cf}$ & 13 \\
\hline La Soledad & $1439.3 \mathrm{def}$ & $16.6 \mathrm{bcd}$ & 12 & $13.6 \mathrm{bcd}$ & 11 & $3.9 \mathrm{ab}$ & 2 & $177.7 \mathrm{fg}$ & 13 & $7.9 \mathrm{de}$ & 13 & $6.5 \mathrm{cde}$ & 11 & $3.5 \mathrm{a}$ & 4 & $1.1 \mathrm{ef}$ & 13 \\
\hline Montana Sumpul & 1418.7 ef & $16.4 \mathrm{~cd}$ & 14 & $13.2 \mathrm{~cd}$ & 13 & $3.7 \mathrm{abc}$ & 9 & $182.8 \mathrm{efg}$ & 12 & $8.2 \mathrm{cde}$ & 11 & $6.4 \mathrm{cde}$ & 13 & $3.4 \mathrm{a}$ & 7 & $1.3 \mathrm{cde}$ & 8 \\
\hline Juquila & $1252.1 \mathrm{f}$ & $16.0 \mathrm{~d}$ & 16 & $12.3 \mathrm{~d}$ & 15 & $4.1 \mathrm{a}$ & 1 & $129.6 \mathrm{gb}$ & 15 & 7.1e & 15 & 5.1ef & 16 & $3.6 \mathrm{a}$ & 1 & $1.1 \mathrm{ef}$ & 13 \\
\hline L as Piedrecitas & $1262.0 \mathrm{f}$ & $16.1 \mathrm{~d}$ & 15 & $11.9 \mathrm{~d}$ & 16 & $3.8 \mathrm{abc}$ & 6 & $117.4 \mathrm{~h}$ & 16 & $7.0 \mathrm{e}$ & 16 & $5.4 \mathrm{f}$ & 15 & $3.4 \mathrm{a}$ & 7 & $0.9 \mathrm{f}$ & 16 \\
\hline Low Elcvation & 1756.4 & 17.7 & & 14.5 & & 3.7 & & 265.4 & & 9.3 & & 7.5 & & 3.4 & & 1.5 & \\
\hline High Elevation & 1468.6 & 16.6 & & 13.3 & & 3.8 & & 183.8 & & 8.0 & & 6.4 & & 3.5 & & 1.2 & \\
\hline
\end{tabular}

Means in a column followed by a common letter do not differ significantly at $5 \%$; rk $=$ rank.

Low Elevation and High Elevation = Low and High Elevation P. tecunumanii.

Table 8. - Means of growth and stem straightness of $P$. tecunumanii provenances at Maswera.

\begin{tabular}{|c|c|c|c|c|c|c|c|c|c|c|c|c|c|c|c|c|c|}
\hline \multirow[t]{2}{*}{ Provenance } & \multicolumn{17}{|c|}{ Means } \\
\hline & $\begin{array}{l}\text { vol8 } \\
\left(\mathrm{dm}^{3}\right)\end{array}$ & $\begin{array}{l}\mathrm{dbh} 8 \\
(\mathrm{~cm})\end{array}$ & rk & $\begin{array}{l}\text { hgl } 8 \\
\text { (m) }\end{array}$ & rk & $\begin{array}{l}\text { str8 } \\
(1-7)\end{array}$ & rk & $\begin{array}{l}\text { vol5 } \\
\left(\mathrm{dm}^{3}\right)\end{array}$ & rk & $\begin{array}{r}\text { dbh5 } \\
(\mathrm{cm})\end{array}$ & rk & $\begin{array}{l}\text { hgl5 } \\
\text { (m) }\end{array}$ & rk & $\begin{array}{l}\text { str5 } \\
(1-7)\end{array}$ & rk & $\begin{array}{l}\text { hgl2 } \\
\text { (m) }\end{array}$ & rk \\
\hline Villa Santa & $890.6 \mathrm{a}$ & $13.8 \mathrm{a}$ & 1 & $12.1 \mathrm{ab}$ & 1 & $4.3 \mathrm{a}$ & 2 & $138.3 \mathrm{a}$ & 2 & $7.1 \mathrm{ab}$ & 2 & $6.5 \mathrm{ab}$ & 2 & $3.2 a b c$ & 3 & $1.1 \mathrm{~b}$ & 3 \\
\hline San Francisco & $873.1 \mathrm{ab}$ & $13.6 \mathrm{a}$ & 2 & $12.2 \mathrm{a}$ & 2 & $4.2 \mathrm{a}$ & 5 & $157.9 \mathrm{a}$ & 1 & $7.6 \mathrm{a}$ & 1 & $6.9 a$ & 1 & $3.6 \mathrm{a}$ & 1 & $1.5 \mathrm{a}$ & 1 \\
\hline Jocón & $762.6 \mathrm{abc}$ & $12.9 \mathrm{ab}$ & 4 & $11.5 \mathrm{abc}$ & 3 & $4.3 a$ & 2 & $93.6 \mathrm{bc}$ & 6 & $6.1 \mathrm{bc}$ & 6 & $5.6 \mathrm{be}$ & 7 & $3.1 \mathrm{abc}$ & 5 & $1.0 \mathrm{cb}$ & 5 \\
\hline Cusuco & 727.5abcd & $12.7 \mathrm{ab}$ & 5 & $11.2 \mathrm{abc}$ & 5 & $4.4 a$ & 1 & $100.5 b$ & 4 & $6.2 \mathrm{bc}$ & 4 & $5.7 \mathrm{bc}$ & 6 & $3.2 \mathrm{abc}$ & 3 & $1.0 \mathrm{cb}$ & 5 \\
\hline Mt Pine Ridge & 711.7abcde & 13. lab & 3 & 11.0abed & 6 & $3.9 \mathrm{a}$ & 13 & $96.2 b c$ & 5 & $6.3 \mathrm{bc}$ & 3 & $5.8 \mathrm{bc}$ & 3 & 3.3ab & 2 & $1.2 \mathrm{~b}$ & 2 \\
\hline San Estaban & 702.4abcde & $12.5 \mathrm{abc}$ & 6 & 11.3abc & 4 & $4.1 \mathbf{a}$ & 7 & $102.5 \mathrm{~b}$ & 3 & $6.1 \mathrm{bc}$ & 6 & $5.8 \mathrm{bc}$ & 3 & $3.0 \mathrm{abc}$ & 8 & $1.0 \mathrm{cb}$ & 5 \\
\hline Culmi & $658.7 \mathrm{cde}$ & $12.2 \mathrm{abcd}$ & 7 & $10.6 a b c d e$ & 8 & $4.0 \mathrm{a}$ & 11 & $89.4 b c d$ & 7 & $5.8 \mathrm{cde}$ & 9 & $5.6 \mathrm{bc}$ & 7 & $3.1 a b c$ & 5 & $0.9 \mathrm{~cd}$ & 8 \\
\hline Montana Sumpul & $636.8 \mathrm{cdef}$ & 12.0acd & 9 & 10.7abcde & 7 & $4.3 \mathrm{a}$ & 2 & $87.1 \mathrm{bcd}$ & 8 & $6.0 \mathrm{bcde}$ & 8 & $5.4 \mathrm{~cd}$ & 9 & $3,1 \mathrm{abc}$ & 5 & $0.9 \mathrm{~cd}$ & 8 \\
\hline Yucul & $610.5 \mathrm{cdef}$ & 12. labed & 8 & $10.6 \mathrm{abcde}$ & 8 & $4.2 a$ & 5 & $80.5 h$ & 9 & $6.2 \mathrm{bc}$ & 4 & $5.8 \mathrm{bc}$ & 3 & $3.0 \mathrm{abc}$ & 8 & $1.1 \mathrm{~b}$ & 3 \\
\hline $\mathrm{LaPa}$ & $568.5 \mathrm{cdelg}$ & $11.1 \mathrm{bcd}$ & 12 & $10.2 \mathrm{abcde}$ & 10 & $4.0 \mathrm{a}$ & 11 & $76.9 \mathrm{bcde}$ & 10 & $5.3 \mathrm{cdefg}$ & 11 & $5.1 \mathrm{~cd}$ & 10 & $2.8 \mathrm{bc}$ & 11 & $0.9 \mathrm{~cd}$ & 8 \\
\hline San Jerónimo & $568.4 \mathrm{cdefg}$ & $11.7 \mathrm{abed}$ & 10 & 10.1bede & 11 & 4. $1 \mathbf{a}$ & 7 & $72.2 \mathrm{bcde}$ & 11 & $5.5 \mathrm{cdef}$ & 10 & $5.1 \mathrm{~cd}$ & 10 & $3.0 \mathrm{abc}$ & 8 & $0.8 \mathrm{de}$ & 12 \\
\hline Las Victorias & $556.6 \mathrm{defg}$ & $11.2 \mathrm{bed}$ & 11 & $9.9 \mathrm{cde}$ & 12 & $3.9 a$ & 13 & $59.9 \mathrm{cdel}^{\circ}$ & 13 & $4.9 \mathrm{defg}$ & 13 & $4.9 \mathrm{cde}$ & 12 & $2.8 \mathrm{bc}$ & 11 & $0.9 \mathrm{~cd}$ & 8 \\
\hline La Soledad & $518.1 \mathrm{efg}$ & $11.1 \mathrm{bed}$ & 12 & $9.8 \mathrm{cde}$ & 13 & $4.1 \mathrm{a}$ & 7 & $66.5 \mathrm{def}$ & 12 & $5.2 \mathrm{cdfg}$ & 12 & $4.9 \mathrm{cde}$ & 12 & $2.7 \mathrm{bc}$ & 13 & $0.8 \mathrm{de}$ & 12 \\
\hline Guajiquiro & $442.7 \mathrm{lg}$ & $10.5 \mathrm{~cd}$ & 14 & $9.2 \mathrm{de}$ & 14 & $3.8 \mathbf{a}$ & 16 & $51.1 \mathrm{ef}$ & 14 & $4.61 \mathrm{~g}$ & 15 & $4.5 \mathrm{de}$ & 14 & $2.6 c$ & 16 & $0.7 \mathrm{e}$ & 14 \\
\hline Las Piedrecitas & $398.4 \mathrm{fg}$ & $10.2 \mathrm{~d}$ & 16 & $8.7 \mathrm{e}$ & 16 & $3.9 \mathrm{a}$ & 13 & $37.7 \mathrm{f}$ & 16 & $4.1 \mathrm{~g}$ & 16 & $3.9 \mathrm{e}$ & 16 & $2.7 \mathrm{bc}$ & 13 & $0.6 \mathrm{e}$ & 16 \\
\hline Juquila & $391.0 \mathrm{~g}$ & $10.3 \mathrm{~d}$ & 15 & $8.8 \mathrm{e}$ & 15 & $4.1 \mathbf{a}$ & 7 & $50.0 \mathrm{ef}$ & 15 & $4.8 \mathrm{efg}$ & 14 & $4.5 \mathrm{de}$ & 14 & $2.7 \mathrm{bc}$ & 13 & $0.7 \mathrm{e}$ & 14 \\
\hline Low Elevation & 720.7 & 12.7 & & 11.1 & & 4.1 & & 104.8 & & 6.3 & & 6.3 & & 3.1 & & 1.1 & \\
\hline
\end{tabular}

Means in a column followed by a common letter do not differ significantly at $5 \%$; rk $=$ rank.

Low Elevation and High Elevation = Low and High Elevation P. tecunumanii. 
Table 9. - Family ranges of two-, five- and eight-year growth and stem straightness traits for LE and HE $P$. tecunumanii at individual sites.

\begin{tabular}{|c|c|c|c|c|c|c|c|c|c|c|c|}
\hline \multirow[t]{3}{*}{ Site } & \multirow{3}{*}{$\begin{array}{l}P . \\
\text { tecunumanii }\end{array}$} & \multirow[t]{3}{*}{ Range } & \multicolumn{9}{|c|}{ Trait } \\
\hline & & & hgt2 & hgt 5 & hgt8 & dbh5 & dbh 8 & vol5 & vol8 & str5 & str8 \\
\hline & & & $(\mathrm{m})$ & (m) & (m) & (cm) & $(\mathrm{cm})$ & $\left(\mathrm{dm}^{3}\right)$ & $\left(\mathrm{dm}^{3}\right)$ & $(1-7)$ & $(1-7)$ \\
\hline \multirow[t]{4}{*}{ Stapleford } & LE & from & 1.00 & 5.70 & 10.41 & 11.0 & 15.3 & 254.57 & 1031.66 & 2.5 & 2.7 \\
\hline & & to & 1.90 & 8.51 & 13.72 & 14.5 & 20.6 & 500.82 & 2099.33 & 3.7 & 4.3 \\
\hline & $\mathrm{HE}$ & from & 0.89 & 5.94 & 10.42 & 8.8 & 21.9 & 277.62 & 1093.51 & 3.0 & 3.0 \\
\hline & & to & 1.54 & 8.95 & 14.61 & 13.6 & 15.5 & 602.00 & 2581.54 & 4.1 & 4.9 \\
\hline \multirow[t]{4}{*}{ Gungunyana } & $\mathrm{LE}$ & from & 0.99 & 6.34 & 12,43 & 7.4 & 15.5 & 141.80 & 1220.34 & 3.0 & 2.8 \\
\hline & & to & 2.75 & 8.72 & 16.46 & 11,4 & 19.7 & 440.08 & 2297.86 & 4.0 & 4.3 \\
\hline & $\mathrm{HE}$ & from & 0.70 & 4.54 & 9.44 & 5.4 & 11.6 & 62.44 & 589.23 & 2.7 & 2.7 \\
\hline & & to & 1.87 & 8.49 & 15.82 & 11,0 & 20.4 & 388.50 & 2400.56 & 4.0 & 4.4 \\
\hline \multirow[t]{4}{*}{ Cashel } & LE & from & 0.90 & 5.74 & 9.43 & 7.1 & 13.6 & 126.83 & 724.12 & 2.8 & 2.2 \\
\hline & & to & 2.02 & 7.71 & 12.63 & 11.3 & 18.5 & 362.43 & 1558.7 & 4.0 & 3.8 \\
\hline & $\mathrm{HE}$ & from & 0.64 & 4.27 & 7.82 & 5.0 & 10.2 & 76.38 & 526.80 & 2.6 & 2.7 \\
\hline & & to & 1.69 & 7.46 & 12,44 & 10.3 & 19.2 & 291.92 & 1692.52 & 4.6 & 4.1 \\
\hline \multirow[t]{4}{*}{ Maswera } & LE & from & 0.64 & 4.32 & 8.65 & 4.0 & 9.6 & 39.80 & 383.95 & 2.4 & 3.3 \\
\hline & & to & 1.70 & 7.52 & 13.38 & 8.5 & 15.5 & 206.22 & 1189.95 & 3.0 & 4.8 \\
\hline & $\mathrm{HE}$ & from & 0.42 & 3.36 & 7.22 & 3.3 & 7.2 & 22.18 & 241.06 & 1.8 & 2.8 \\
\hline & & to & 1.21 & 6.55 & 12.34 & 7.5 & 14.7 & 150.55 & 1054.44 & 3.6 & 5.0 \\
\hline
\end{tabular}

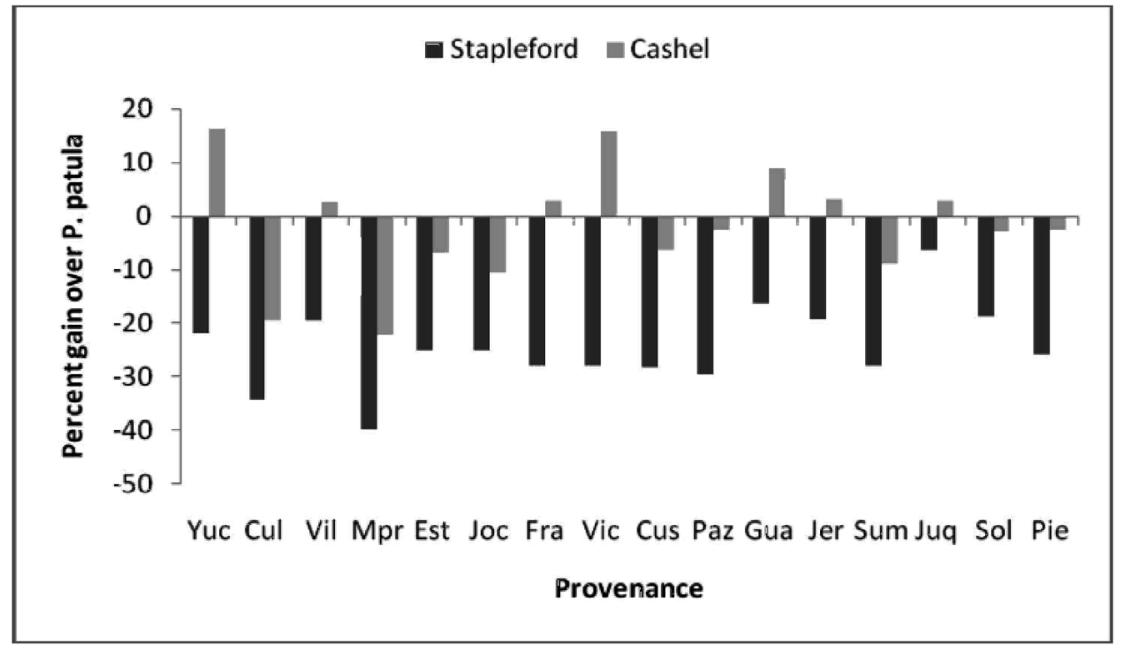

(a)

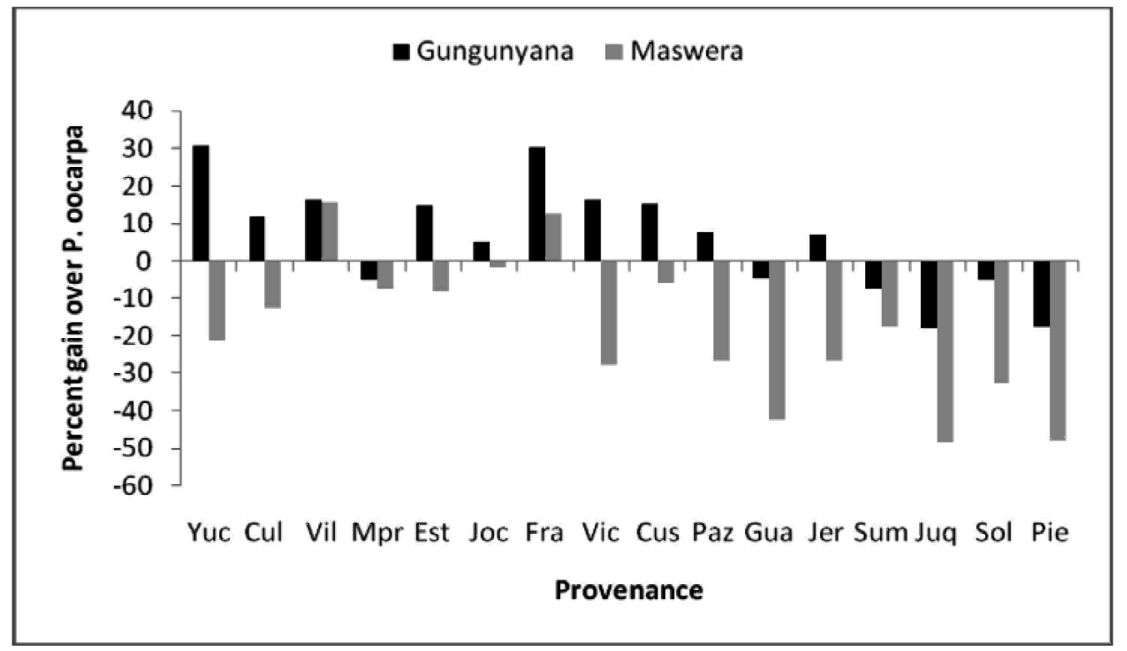

(b)

Figure 1. - Advantage (gain) in eight-year volume from using seed of P. tecunumanii provenances over P. patula (a) at Stapleford and Cashel and over P. oocarpa (b) at Gungunyana and Maswera (Provenance codes are shown in Table 1). 
ranked provenances for two-year height at most of the sites but ended among the poorest provenances at age eight years. For eight-year volume, the most productive provenances were Juquila and Guajiquiro at Stapleford, Yucul and Las Victorias at Cashel, San Francisco and Yucul at Gungunyana and Villa Santa and San Francisco at Maswera. The mean annual increments of the most productive provenances at the different sites translated to $26.1 \mathrm{~m}^{3} \mathrm{ha}^{-1} \mathrm{yr}^{-1}$ (1760 m a.s.l.); $17.3 \mathrm{~m}^{3} \mathrm{ha}^{-1} \mathrm{yr}^{-1}$ (1450 m a.s.l.); $24.9 \mathrm{~m}^{3} \mathrm{ha}^{-1} \mathrm{yr}^{-1}$ (1050 m a.s.l.) and $11.1 \mathrm{~m}^{3} \mathrm{ha}^{-1} \mathrm{yr}^{-1}$ (780 $\mathrm{m}$ a.s.l.)

The HE $P$. tecunumanii had its highest eight-year mean individual tree volume of $1729.2 \mathrm{dm}^{3}$ at Stapleford while the LE $P$. tecunumanii had its highest mean volume of $1756.4 \mathrm{dm}^{3}$ at Gungunyana. The stem straightness rating of the HE $P$. tecunumanii was consistently better than that of the LE P. tecunumanii at most of the sites. HE $P$. tecunumanii provenances with consistently superior stem straightness rating included Juquila, San Jeronimo and Las Piedrecitas, while Yucul had superior stem straightness rating among the LE $P$. tecunumanii provenances.

Figure 1 shows the advantage to be gained in eightyear volume from using seed from each of the 16 $P$. tecunumanii provenances over $P$. patula, the currently planted species at Stapleford and Cashel, and over $P$. oocarpa at Gungunyana and Maswera. Commercially deploying seed of any of the $16 P$. tecunumanii provenances instead of $P$. patula would be a disadvantage at the present moment as this will result in negative gains ranging from $-40 \%$ for Mt Pine Ridge to $-6 \%$ for Juquila at Stapleford (Fig. 1a). At Cashel however, some provenances were significantly superior to $P$. patula and their planting in place of $P$. patula would result in gains

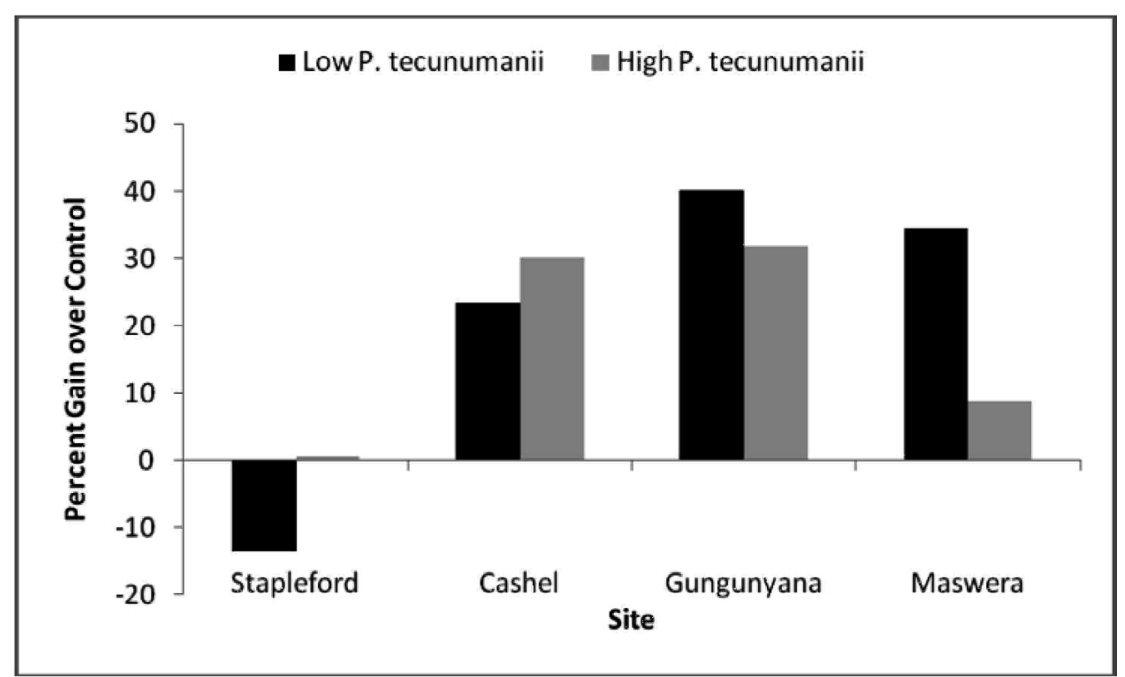

Figure 2. - Advantage (gain) in 8-year volume from using seed from the best 10 families of high and low elevation $P$. tecunumanii over $P$. patula (at Stapleford and Cashel) and over P. oocarpa (at Gungunyana and Maswera).

Table 10. - ANOVA F-values for two-, five- and eight-year height, diameter, volume and stem straightness (hgt2, hgt5, hgt8, dbh5, dbh8, vol5, vol8, str5 and str8) across sites.

\begin{tabular}{|c|c|c|c|c|c|c|c|c|c|c|}
\hline \multirow{2}{*}{ Source } & \multirow{2}{*}{ DF } & \multicolumn{9}{|c|}{ Trait } \\
\hline & & hgt2 & hgt5 & dbh5 & vol5 & str5 & hgt8 & dbh8 & vol 8 & str8 \\
\hline Site S & 3 & $274.6^{* * *}$ & $502.9^{* * * *}$ & $1316.4^{* * * *}$ & $1017.2^{* * *}$ & $210.8^{* * *}$ & $316.2^{* * *}$ & $726.7^{* * *}$ & $524.9^{* * *}$ & $65.9^{* * *}$ \\
\hline Block-within-site B(S) & 16 & & & & & & & & & \\
\hline P. tecunumanii type A & 1 & $124.5^{* * *}$ & $77.96^{* * *}$ & $55.91^{* * *}$ & $25.55^{* * *}$ & $0.12^{\mathrm{ms}}$ & $57.04^{* * *}$ & $28.02^{* * *}$ & $15.90^{* * *}$ & $6.20^{*}$ \\
\hline Site*P. tecunumanii type $\mathrm{S} * \mathrm{~A}$ & 3 & $14.33^{* * *}$ & $57.00^{* * *}$ & $32.98^{* * *}$ & $31.92^{* * *}$ & $50.13^{* * *}$ & $49.14^{* * *}$ & $37.74^{* * *}$ & $36.78^{* * *}$ & $53.72^{* * *}$ \\
\hline $\mathrm{B}(\mathrm{S})^{*} \mathrm{~A}$ & 16 & & & & & & & & & \\
\hline Provenance $\mathrm{P}(\mathrm{A})$ & 14 & $11.87^{* * *}$ & $7.52^{* * *}$ & $7.49^{* * *}$ & $6.08^{* * *}$ & $5.01^{*+*+}$ & $8.07^{* * *}$ & $4.83^{* * *}$ & $5.03^{*+*}$ & $5.93^{* * *}$ \\
\hline LE $P$. tecunumanii provenance & 7 & $14.47^{* * *}$ & $8.19^{* * * *}$ & $8.54^{* * * *}$ & $8.12^{* * *}$ & $4.32^{* * *}$ & $8.45^{* * *}$ & $5.73^{* * 3}$ & $7.53^{\text {**** }}$ & $5.62^{* * *}$ \\
\hline HE $P$. tecunumanii provenance & 7 & $9.62^{* * *}$ & $8.48^{* * *}$ & $6.85^{* * *}$ & $4.27^{* * *}$ & $5.45^{*+*}$ & $7.86^{* * *}$ & $4.26^{* * 3}$ & $3.27^{* *}$ & $6.17^{* * *}$ \\
\hline $\mathrm{S}^{*} \mathrm{P}(\mathrm{A})$ & 42 & $6.58^{k+k}$ & $9.36^{* * * *}$ & $7.02^{* * * k}$ & $7.42^{* * *}$ & $6.38^{* * *}$ & $7.35^{*+*}$ & $5.09^{4 * *}$ & $5.43^{\text {*** }}$ & $5.14^{* * *}$ \\
\hline S*LE $P$. tecunumanii & 21 & $6.83^{* * * 5}$ & $9.81^{* * *}$ & $7.25^{* * *}$ & $8.76^{* * *}$ & $5.21^{* * *}$ & $6.06^{* * *}$ & $4.55^{* * *}$ & $4.24^{* * * *}$ & $4.04^{* * *}$ \\
\hline $\mathrm{S} * \mathrm{HE} P$ tecunumanii & 21 & $5.64^{* * *}$ & $9.06^{* * *}$ & $6.87^{* * *}$ & $6.18^{* * *}$ & $7.01^{* * *}$ & $8.11^{* * *}$ & $5.36^{* * *}$ & $6.30^{k * *}$ & $5.66^{* * *}$ \\
\hline $\mathrm{B}(\mathrm{S}) * \mathrm{P}(\mathrm{A})$ & 224 & & & & & & & & & \\
\hline Family(PA) & 144 & $5.21^{* * *}$ & $3.53^{* * *}$ & $3.71^{* * * *}$ & $2.86^{* * *}$ & $2.36^{* * *}$ & $2.98^{* * *}$ & $3.53^{* * z}$ & $3.05^{\kappa * * *}$ & $2.38^{* * *}$ \\
\hline Family-within-LE $P$. tecumumanii $) \mathrm{F}(\mathrm{P})$ & 72 & $4.28^{* * *}$ & $2.33^{* * * *}$ & $2.68^{* * * *}$ & $2.47^{* * *}$ & $1.87^{*+*}$ & $1.97^{* * *}$ & $2.67^{* * *}$ & $2.43^{*+*}$ & $2.36^{* * *}$ \\
\hline Family-within-HE $P$. tecunumanii) $\mathbf{F}(\mathrm{P})$ & 72 & $6.62^{* * *}$ & $4.96^{* * *}$ & $4.86^{* * *}$ & $3.33^{* * *}$ & $2.83^{* * * *}$ & $4.07^{* * *}$ & $4.46^{* * 8}$ & $3.77^{* * *}$ & $2.39^{* * *}$ \\
\hline $\mathrm{S}^{*} \mathrm{~F}(\mathrm{PA})$ & 432 & $1.65^{* * * *}$ & $1.55^{* * *}$ & $1.65^{* * *+}$ & $1.52^{* * *}$ & $1.57^{* * *+}$ & $1.65^{* * *}$ & $1.75^{* * *}$ & $1.53^{*+*}$ & $1.53^{* * * *}$ \\
\hline Site*F(LE $P$. tecunumanii $)$ & 216 & $1.56^{* * *}$ & $1.14^{\mathrm{ns}}$ & $1.25^{* *}$ & $1.35^{* *}$ & $1.10^{\mathrm{ns}}$ & $1.19^{*}$ & $1.12^{\mathrm{ns}}$ & $1.19^{*}$ & $1.13^{\text {ns }}$ \\
\hline Site ${ }^{*} \mathrm{~F}(\mathrm{HE} P$. tecunumanii $)$ & 216 & $1.80^{* * *}$ & $2.03^{*+*}$ & $2.11^{* * * *}$ & $1.72^{* *}$ & $2.04^{* *+*}$ & $2.14^{* * *}$ & $2.42^{* * 3}$ & $1.94^{* * * *}$ & $1.87^{* * *}$ \\
\hline Error & 1152 & & & & & & & & & \\
\hline
\end{tabular}

$\mathrm{ns}, *, * * *$ and $* * * *=$ not significant at 5 , significant at $5,1 \%$ and $0.1 \%$ respectively. 
as high as $16 \%$. The planting of any of the 16 $P$. tecunumanii provenances at Gungunyana and Maswera instead of $P$. oocarpa, the appropriate control for the lower, warmer and drier sites would result in eight-year volume gains ranging from $-18 \%$ to $31 \%$ at Gungunyana and from $-48 \%$ to $16 \%$ at Maswera respectively (Fig. 1b). At these two sites, most of the negative gains were associated with $\mathrm{HE} P$. tecunumanii provenances.

The seed of $P$. tecunumanii was collected and tested as family seedlots, and therefore permitted the estimation of potential gains that would result from using seed of selected families instead of bulk seed of provenances. Planting seed from the top 10 ranked families from the HE $P$. tecunumanii gave yield advantage in eight-year volume of $1 \%$ and $22 \%$ over P. patula at Stapleford and Cashel, and $33 \%$ and $10 \%$ over P. oocarpa at Gungunyana and Maswera respectively (Fig. 2). The planting of seed from the top 10 families of the LE P. tecunumanii gave gains in eight-year volume of respectively $-13 \%$ and $23 \%$ over P. patula at Stapleford and Cashel, and $40 \%$ and $34 \%$ over $P$. oocarpa at Gungunyana and Maswera (Fig. 2).

The combined analysis of all the data across sites revealed significant effects $(\mathrm{P}<0.001)$ of site, elevation $P$. tecunumanii type, provenance and family and their associated interactions for all the traits (Table 10). The interaction between site and $P$. tecunumanii type was mostly a change in rank between sites, with the $\mathrm{HE}$ $P$. tecunumanii being superior to the LE $P$. tecunumanii at higher altitude sites and vice versa at lower altitude sites. Families of the HE $P$. tecunumanii were more interactive with sites compared to families of the LE P. tecunumanii (Table 10).

\section{Discussion and Conclusions}

The study revealed substantial differences between the LE and HE $P$. tecunumanii in adaptation, growth and stem quality and the differences depended on age of growth and also on site. The differences between the two types of $P$. tecunumanii were very large and possibly exaggerated at lower and drier altitudes where the LE $P$. tecunumanii was significantly superior in growth and adaptation and this was attributed to maladaptation of some of the provenances of the HE $P$. tecunumanii such as Las Piedrecitas that occurs in a cloud forest environment at $2200 \mathrm{~m}$ in natural stands and may be drought sensitive. In well watered environments the LE $P$. tecunumanii had a higher early height growth which however did not persist beyond the fifth year of growth. Although there are not many well designed studies comparing the performance of the LE and $\mathrm{HE}$ $P$. tecunumanii together, the study by HoDGE and DvorAK (1999) of the two population groups based on some contiguous trials gave a pointer. In one such test in Colombia, established at $1750 \mathrm{~m}$ a.s.l., the $\mathrm{HE}$ $P$. tecunumanii had an average volume of $2518 \mathrm{dm}^{3}$ compared to $2282 \mathrm{dm}^{3}$ for the LE $P$. tecunumanii at eight years (HoDGE and DvorAK, 1999). These figures when contrasted with results of this study of eight-year volume of $1729.2 \mathrm{dm}^{3}$ and $1588.6 \mathrm{dm}^{3}$ for the $\mathrm{HE}$ and $\mathrm{LE}$
$P$. tecunumanii respectively at Stapleford, which although lower, largely confirm that the $\mathrm{HE}$ $P$. tecunumanii is more productive than the LE $P$. tecunumanii at high altitudes. In the present study, at medium altitude (1450 $\mathrm{m}$ a.s.l.), the $\mathrm{LE}$ and $\mathrm{HE}$ $P$. tecunumanii were comparable in productivity (1155.1 versus $1174.5 \mathrm{dm}^{3}$ ) respectively, which is also consistent with the results from South Africa at a comparable altitude (HodGe and DvoraK, 1999). At lower altitudes, the results of the present study showed that the LE $P$. tecunumanii is superior in growth to the $\mathrm{HE}$ $P$. tecunumanii and is largely consistent with the results of Hodge and DvorAK (1999), who reported that the LE $P$. tecunumanii was superior to the HE P. tecunumanii. The fact that, the LE $P$. tecunumanii outperformed the HE $P$. tecunumanii at lower altitudes, and vice versa, implies that a need probably exists, to deploy seed of their provenances in the same pattern in afforestation programmes to optimise yields.

In this study, the Mt Pine Ridge provenance was found to be significantly different from the other LE $P$. tecunumanii provenances. The provenance showed exceptional height growth at two years, where it was ranked among the top but its growth rate subsequently declined with increasing age, to be ranked lowest for both growth and stem straightness at age five and eight years. ZAMUDIO (1992) studied rank changes in $P$. tecunumanii, and observed that most family rank changes in growth occur in the first 3 years of growth. Such early fast growth could be exploited in situations where weeds are a serious problem and seed sources that quickly escape or suppress weed growth will reduce the frequency of weeding. In the case of Mt Pine Ridge provenance, its advantage of early fast growth could be far outweighed by its relatively poor growth beyond the post establishment phase as well as its poor stem form which would result in reduced plantation productivity. The poor stem straightness rating exhibited by the Mt Pine Ridge provenance in these tests is consistent with the observations made by DvorAK (1986), who proposed to separate Belize sources from the other LE $P$. tecunumanii because of the preponderance of poor stem form in the natural stands.

The Mexican provenances comprising Juquila and Las Piedrecitas, which are also isolated from the other HE $P$. tecunumanii populations were also found to be significantly different in growth and stem straightness from the other HE sources. At low altitude sites (Gungunyana and Maswera), the observed differences could have been exaggerated as a result of maladaptation of these (Juquila and Las Piedrecitas) provenances which originate from cooler and well watered environments while the differences at the higher altitude (Stapleford) could have been a result of the exceptionally high growth rate of Juquila, which is now regarded as not $P$. tecunumanii (DvoraK and RAYMond, 1991; Hodge and DvoraK, 1999; DvorAK, 2008). The Juquila provenance has been shown to contain markers that align it with Pinus herrerae or closely related $P$. pringlei and was recently described as P. herrerae (DvORAK et al., 2000b; DvORAK et al., 2007), Some of the largest trees with imposing stem form and fine branching in these tests were from this provenance. 
Las Piedrecitas was only average in volume production in the present study and was also found to be a poor provenance in tests in Brazil (MouRA and DvoRAK, 1998).

Although no individual provenance of $P$. tecunumanii was superior to the currently grown second generation bred $P$. patula at high altitude, some families of these $P$. tecunumanii provenances, particularly those from the HE $P$. tecunumanii were marginally superior by about 0.6 percent at high altitude and by up to 21 percent at medium altitude (1450 $\mathrm{m}$ a.s.1.), the lower limit of tolerance for economic production of $P$. patula in Zimbabwe. $P$. tecunumanii provenances and families have been tested against $P$. patula in a number of trials and results have been variable (MoRris, 1988; DvORAK and SHAw, 1992). The variable results may be a result of different ages of the tests and appropriateness of the controls. This is consistent with the present results in which $P$. patula was outperformed by a number of $P$. tecunumanii provenances at age two years in its recommended altitudinal range (above $1760 \mathrm{~m}$ a.s.l.) but the latter was outperformed from age five years. Also, in some of the studies cited, $P$. patula may have been inappropriately used as a control species in for example, warm low altitudes where its performance is known to be poor. Caution is required in the interpretation of the comparisons between $P$. tecunumanii and $P$. patula or $P$. oocarpa as the seed of these controls were all improved seed. First generation bred P. patula, for example, is known to have a volume yield advantage over unimproved material of more than 17.5 percent (BARNES, 1977) realised from the intense breeding and selection, while second generation bred seed is believed to have an improvement in volume of up to 30 percent. If these improvements were discounted the current conclusions could probably be altered.

In the lower warmer and drier sites, the use of seed from $P$. tecunumanii provenances such as Yucul and San Francisco or seed from selected superior families in afforestation instead of $P$. oocarpa could substantially increase timber yields. The superiority of some provenances of $P$. tecunumanii over $P$. oocarpa has in the past been demonstrated in Zimbabwe (CROCKFORD et al., 1988; NYOKA and BARNES, 1995); in Brazil (MURILlO and DvorAK, 1988; Moura et al., 1991 cited by MourA and DvorAK, 1998); in South Africa (DvorAK and SHAW, 1992) and in Kenya (WRIGHT et al., 1992). Overall, some of the most productive provenances such as San Jerónimo, Villa Santa, Yucul, Guajiquiro and Juquila (although now regarded Pinus herrerae) identified in this study have shown superiority in other studies (DvoRAK and SHAW, 1992; WRIGHT and OsORIO, 1992; NYOKA and BARNES, 1995; Moura and DvoraK, 1998; HodGe and DVORAK, 1999).

In this study, genotype-environment interaction was found to be present and significant at three levels namely elevation $P$. tecunumanii type, provenance and at family level. The LE $P$. tecunumanii was found to be more productive in lower altitude environments while the HE $P$. tecunumanii was found to be more productive in high altitudes. This study revealed that site*family interaction is present and probably of practical importance in the HE sources but is less apparent in the LE sources. Significant site*provenance and site*family interactions have also been reported in $P$. tecunumanii (DvoraK et al., 1989; DVORAK and Ross, 1994; CrockFORD et al., 1990; HodGE and DvorAK, 1999). DvORAK and SHAW (1992) found moderate site*family interaction and very little site* provenance interaction.

The most productive provenances by environment were identified as Juquila and Guajiquiro $(1760 \mathrm{~m}$ a.s.l.), Yucul and Las Victorias (1450 m a.s.l.), San Francisco and Yucul (1050 m a.s.l) and Villa Santa and San Francisco (780 m a.s.l.). The eight-year timber yield potential of the LE $P$. tecunumanii at lower altitude (1050 m a.s.l.) was found to be similar to that of the HE $P$. tecunumanii at higher altitude $\left(1760 \mathrm{~m}\right.$ a.s.l). The $2^{\text {nd }}$ generation bred clonal seed of $P$. patula was found to be superior to all the provenances of $P$. tecunumanii although the most productive families of the latter were comparable in growth. A number of provenances of $P$. tecunumanii were also shown to be significantly superior to first generation bred clonal seed source of $P$. oocarpa in the medium and lower altitude sites, presenting opportunities for immediate deployment of seed of $P$. tecunumanii in these environments. Furthermore, the LE $P$. tecunumanii has been shown to be highly resistant to pitch canker compared to the $\mathrm{HE}$ or the widely planted $P$. patula (DvorAK et al., 2009), again presenting opportunities for its immediate deployment in areas that are affected by the disease. There is however, no immediate yield advantage of using $P$. tecunumanii seed in high altitude environments currently planted to $P$. patula, unless the intention is for species diversity or replacing the highly invasive $P$. patula. Considering that some of the families of $P$. tecunumanii were comparable in growth to second generation bred $P$. patula, breeding and selection could also hasten the planting of $P$. tecunumanii in the higher altitudes in the near future. The real advantage of using $P$. tecunumanii versus $P$. patula will not be realized until the $P$. tecunumanii is taken through one more generation of breeding.

\section{Acknowledgements}

The material used in this study was supplied by the Oxford Forestry Institute, for which we are grateful. We are also grateful to the Station Managers of John Meikle, Muguzo and Gungunyana Research Stations, Messrs T. MAngezi, H. C. MHongweH and J. Garigayi for establishing, maintaining and assessing the trials. The late Dr R. D. BARNES of the Oxford Forestry Institute, Oxford University gave guidance in the design, management and assessment of these trials.

\section{References}

BARNes, R. D. (1977): Population improvement through selection and hybridization in Pinus patula, P. elliottii and P. taeda in southern Africa. In: Third World Consultation on Forest Tree Breeding. Canberra, Australia, 21-26 March 1977. CSIRO. FO-FTB-77 3/3: 489-505. 
BARNES, R. D. and G. L. GiBson (1986): A method to assess stem straightness in tropical pines. Commonwealth Forestry Review 65: 168-171.

BIRKS, J. S. and R. D. BARNES (1990): Provenance variation in Pinus caribaea, $P$. oocarpa and P. patula ssp. tecunumanii. Tropical Forestry Papers. No. 21, 40 pp.

Crockford, K. J., Birks, J. S. and R. D. Barnes (1988): Family within provenance trials of Pinus caribaea, $P$. oocarpa and P. patula ssp. tecunumanii: Early results and implication for breeding strategy. In: GIBSON, G. L., Griffin, A. R. and Matheson, A. C. Eds. Populations structure and genetic improvement strategies in clonal and seedling forestry. Proceedings of IUFRO conference, Pattaya, Thailand. November 1988. pp 176-183.

Crockford, K. J., A. J. Dunsdon, W. B. H. Baylis, J. S. BIRKS and R. D. BARNES (1990): Evaluation of Tropical Pine provenance and progeny tests. ODA Research Scheme R.4346. Oxford Forestry Institute. University of Oxford. Final Report. 136pp.

Dvorak, W. S. (1986): Provenance/progeny testing of Pinus tecunumanii. In: Proc. of a joint Meeting of Working Parties on Breeding Theory, Progeny Testing and Seed Orchards. Williamsburg, USA. pp 299-309.

Dvorak, W. S., BALOCCHI, C. E. and R. H. RAYMond (1989): Performance and Stability of Provenances and Families of $P$. tecunumanii in the tropics and subtropics. pp 187-196. In: Proceedings of an IUFRO conference on Breeding Tropical Trees: Population Structure and Genetic Improvement Strategies in Clonal and seedling Forestry. Pattaya, Thailand, November, 1988.

DvoraK, W. S and R. H. RAYMOND (1991): The taxonomic status of closely related cone pines in Mexico and Central America. New Forests 4: 291-307.

DvORAK, W. S. and E. A. SHAW (1992): Five year growth results for growth and stem form of Pinus tecunumanii. In Brazil, Colombia and South Africa. CAMCORE bulletin on Tropical Forestry 10. College of forest Resources. North Carolina State University, Raleigh, NC, USA. 22p.

DvorAK, W. S. and K. D. Ross (1994): Three-year growth and stability of Honduran provenances and families of Pinus tecunumanii. Forest Ecology and Management 63: $1-11$.

Dvorak, W. S., G. R. Hodge, E. A. Gutierrez, F. S. Malan and T. K. Stanger (2000a): Pinus tecunumanii. pp 188-209. In: Conservation \& Testing of Tropical \& Subtropical Forest Tree Species by the CAMCORE Cooperative, College of Natural Resources, NCSU, Raleigh, NC. USA.

Dvorak, W. S., J. E. Kietzka, T. K. Stanger and M. MapuLA (2000b): Pinus herrerae. pp 75-84. In: Conservation \& Testing of Tropical \& Subtropical Forest Tree Species by the CAMCORE Cooperative, College of Natural Resources, NCSU, Raleigh, NC. USA.

Dvorak, W. S., E. Kietzka, G. R. Hodge, A. Nel, G. A. dos SANTOS and C. GANTZ (2007): Assessing the potential of Pinus herrerae as a plantation species for the subtropics. Forest Ecology and Management Forest Ecology 242: 598-605.

Dvorak, W. S., K. M. PotTeR, V. D. Hipkins and G. R. Hodge (2009): Genetic diversity and gene exchange in Pinus oocarpa, a Mesoamerican pine with resistance to the pitch canker fungus (Fusarium circinatum). International Journal of Plant Sciences 170: 609-626.

DvorAK, W. S., (2008): Estamos más cerca de entender la ascendencia de las poblaciones de la "variante patula" en la Sierra Madre del sur, México? Foresta Veracruzana 10(1): 59-66.
Eguiluz-Piedra, T. (1986): Taxonomic relationships of Pinus tecunumanii from Guatemala. Commonwealth Forestry Review 65: 303-313.

Furman, B. J., D. Grattapaglia, W. S. DvoraK and D. M. O'MALLEY (1997): Analysis of genetic relationships of Central American and Mexican pines using RAPD markers that distinguish species. Molecular Ecology 6: 321-331.

Grattapaglia, D., D. M. O'Malley and W. S. Dvorak (1993): Phylogenetic analysis of Central American and Mexican pines using RAPD markers as bulked DNA samples. pp 468-473. In: Proc. IUFRO S2.02.08 Conference on Breeding Tropical Trees, edited by C. C. LAMBETH and W. S. DvorAK. Cartagena and Cali, Colombia. 9-18 October 1992.

Hodge, G. R. and W. S. Dvorak (1999): Genetic Parameters and Provenance Variation in Pinus tecunumanii in 78 International Trials. Forest Genetics 6: 157-180.

MorRIs, A. R. (1988): The early growth of Pinus oocarpa and Pinus patula ssp. tecunumanii provenances in the Usutu Forest, Swaziland. pp 377-378. In: Population structure and genetic improvement strategies in clonal and seedling forestry. Proceedings of IUFRO conference, Pattaya, Thailand, edited by G. L. Gibson, A. R. Griffin, and A. C. MATHESON. November 1988.

MourA, V. P. G. and W. S. DvorAK (1998): Provenance and family performance of Pinus tecunumanii at 12 years of age in the Cerrado region of Brazil. Forest Genetics 5: $137-145$.

Murillo, O. and W. S. DvoraK (1988): Stability of Pinus oocarpa provenances across ten environments. pp 379-380. In: Population structure and genetic improvement strategies in clonal and seedling forestry. Proceedings of IUFRO conference, Pattaya, Thailand, edited by G. L. Gibson, A. R. Griffin, and A. C. Matheson. November 1988.

NYOKA, B. I. and R. D. BARNES (1995): Genetic parameters and provenance productivity of Pinus oocarpa and Pinus patula ssp. tecunumanii. South African Forestry Journal 173: 1-7.

SAS InstituTE INC. (1998): SAS/STAT ${ }^{\circledR}$ User's Guide, Release 6.12 Edition. Cary, NC, USA. SAS Institute, Inc.

Squillace, A. E. and J. P. PerRY JR. (1992): Classification of Pinus patula, $P$. tecunumanii, $P$. oocarpa, $P$. caribaea var. hondurensis and related taxonomic entities. Research Paper SE-285. Asheville, NC: US Department of Agriculture, Forest Service, Southeastern Forest experiment Station, $23 \mathrm{pp}$.

StYles, B. T. and P. S. McCarter (1988): The botany, ecology, distribution and conservation status of Pinus patula ssp. tecunumanii in the Republic of Honduras. Ceiba 29: 3-30.

WRIGHT, J. A. and L. OsoRIO (1992): Results of provenance and family within provenance trials of Pinus tecunumanii in Colombia. Forest Ecology and Management 55: 107-116.

Wright, J. A., G. L. Gibson and R. D. BARNES (1992): Variation in stem volume and wood density in provenances of Pinus oocarpa and P. patula ssp. tecunumanii at Nzoia, Kenya. Commonwealth Forestry Review 71: 203-206.

ZAMUdio, F. (1992): Age-related variation in growth characteristics for families of Pinus tecunumanii in South America. MSc Thesis, College of Forest Resources, North Carolina State University, Raleigh, NC, USA. $62 \mathrm{p}$. 